\title{
The Spectral-Element Method in Seismology
}

\author{
Dimitri Komatitsch ${ }^{1}$ \\ Seismological Laboratory, California Institute of Technology, Pasadena, California, USA
}

Seiji Tsuboi

Institute for Research on Earth Evolution, Japan Agency for Marine-Earth Science and Technology, Yokohama, Japan

Jeroen Tromp

Seismological Laboratory, California Institute of Technology, Pasadena, California, USA

We present the main properties of the spectral-element method, which is well suited for numerical calculations of synthetic seismograms for three-dimensional Earth models. The technique is based upon a weak formulation of the equations of motion and combines the flexibility of a finite-element method with the accuracy of a pseudospectral method. The mesh is composed of hexahedral elements and honors the main discontinuities in the Earth model. The displacement vector is expressed in each element in terms of high-degree Lagrange interpolants, and integrals are computed based upon Gauss-Lobatto-Legendre quadrature, which leads to an exactly diagonal mass matrix and therefore drastically simplifies the algorithm. We use a fluid-solid coupling formulation that does not require iterations at the core-mantle or inner-core boundaries. The method is efficiently implemented on parallel computers with distributed memory based upon a message-passing methodology. We present two large-scale simulations for a realistic three-dimensional Earth model computed on the Japanese Earth Simulator at periods of $5 \mathrm{~s}$ and longer.

\section{INTRODUCTION}

The accurate calculation of seismograms in realistic threedimensional (3-D) Earth models represents an ongoing challenge in local, regional, and global seismology. In the past three decades, a wide variety of numerical techniques has been used to address this issue. The most widely used ap-

${ }^{1}$ Now at Department of Geophysics, Université de Pau et des Pays de l'Adour, France

Seismic Earth: Array Analysis of Broadband Seismograms Geophysical Monograph Series 157

Copyright 2005 by the American Geophysical Union 10.1029/156GM13 proach for full-waveform modeling is probably the finite difference method [e.g., Madariaga, 1976; Virieux, 1986], in which one approximates derivatives by differences between adjacent grid points. This approach has been used to calculate the wave field in 3-D local and regional models [e.g., Graves, 1996; Ohminato and Chouet, 1997]. Unfortunately, this classical method suffers from limitations when addressing the complexity of typical 3-D models, such as the presence of surface topography or major discontinuities within the model [e.g., Robertsson, 1996; Ohminato and Chouet, 1997]. Recently developed optimal or compact finite-difference operators have improved this situation [e.g., Zingg et al., 1996; Zingg, 2000]. Methods that resort to more accurate spatial derivative operators, such as spectral and pseudospectral techniques based on global gridding of the model, have also been used to address regional [e.g., Carcione, 1994] and 
global [e.g., Tessmer et al., 1992] seismic wave propagation problems. However, because of the use of global basis functions (polynomial: Chebyshev or Legendre, or harmonic: Fourier), these techniques are limited to smooth media, and numerical noise (i.e., ringing) appears in the presence of sharp discontinuities in the model, such as major interfaces or faults.

Boundary element [e.g., Kawase, 1988] or boundary integral methods [e.g., Sánchez-Sesma and Campillo, 1991] provide a powerful direct way of incorporating topographic variations and interfaces, but are restricted to a finite number of homogeneous regions. In addition, in 3-D the cost of such techniques increases rapidly with numerical resolution, and a truncation threshold often has to be applied, which leads to numerical artefacts in the solution [e.g., Bouchon et al., 1996].

Classical finite-element methods have been successfully applied to the study of wave propagation in 3-D sedimentary basins [e.g., Bao et al., 1998]. These techniques handle previously mentioned difficulties related to the presence of topography or major interfaces by allowing grid boundaries to coincide with major interfaces. However, the spatial discretization itself is often inadequate because of the low polynomial degree used to expand functions within each element, and, in addition, large linear systems have to be solved by approximate, iterative routines, which increases the cost of the calculations and complicates the implementation of the algorithm, in particular on a parallel computer. A low polynomial degree is traditionally used in such techniques because the complexity of the linear system increases with the degree.

The purpose of this article is to give an introduction to the main properties of the spectral-element method (SEM) for seismic wave propagation. The SEM has been used for two decades in computational fluid dynamics [Patera, 1984]. It has more recently been applied to problems related to two-dimensional (2-D) [Cohen et al., 1993; Priolo et al., 1994] and 3-D local or regional [Komatitsch, 1997; Faccioli et al., 1997; Komatitsch and Vilotte, 1998; Seriani, 1998; Komatitsch and Tromp, 1999; Komatitsch et al., 2004; Liu et al., 2004] and global [Chaljub, 2000; Komatitsch and Tromp, 2002a, b; Komatitsch et al., 2002; Chaljub et al., 2003; Komatitsch et al., 2003; Chaljub and Valette, 2004] seismic wave propagation. We introduce the full complexity of the 3-D Earth, i.e., lateral variations in compressionalwave speed, shear-wave speed, and density in the mantle, a 3-D crustal model, anisotropy, ellipticity, surface topography and bathymetry, as well as the effects of the oceans, rotation, and self-gravitation. All of these effects have been benchmarked in previous publications [Komatitsch and Vilotte, 1998; Komatitsch and Tromp, 1999; Komatitsch et al., 2000a, b; Komatitsch and Tromp, 2002a, b]. In this article, we use a simpler fluid-solid coupling method that does not require numerical iterations, based on the recent work of Chaljub and Valette [2004]. We illustrate how the method can be applied to high-resolution simulations of seismic wave propagation in the 3-D Earth on a very large parallel computer: the Earth Simulator at JAMSTEC in Japan.

\section{DESIGN OF THE MESH}

The first, crucial step in the SEM consists of designing a high-quality mesh for the 3-D model, subject to constraints imposed by the required number of grid points per shortest wavelength, the numerical stability condition, and acceptable geometrical distortions of the elements. The mesh is designed once and for all: seismic wave propagation is generally a small deformation problem and therefore one does not need to consider dynamically deforming meshes or dynamic remeshing. This step is very similar to mesh design for general finite-element methods (FEMs), therefore the reader is referred to Zienkiewicz [1977] and Hughes [1987] for a thorough introduction to such techniques.

The model volume $\Omega$ is subdivided into a number of nonoverlapping elements $\Omega_{e}, e=1, \ldots, n_{e}$, such that $\Omega=$ $\cup_{e=1}^{n_{e}} \Omega_{e}$ (Figure 1). In the context of FEMs, various types of elements $\Omega_{e}$ can be used, such as tetrahedra, hexahedra, pyramids, and prisms. In the classical SEM, however, one can only use hexahedra, for reasons that will be explained in Section 3. It is worth mentioning that SEMs can be developed on triangles [e.g., Sherwin and Karniadakis, 1995; Taylor and Wingate, 2000; Komatitsch et al., 2001], but this leads to theoretical complications that are beyond the scope of this article. The basic idea is that on hexahedral elements one can use a tensor product of 1-D basis functions, which in turn gives an exactly diagonal mass matrix, while with tetrahedra the tensorization is lost. As in any FEM, the mesh needs to be geometrically conforming, i.e., the six sides of each hexahedral element must match up exactly with the sides of neighboring elements. Let us also mention that the SEM can be adapted to geometrically non-conforming meshes based on the so-called 'mortar' matching method [e.g., Chaljub, 2000; Chaljub et al., 2003], and that such non-conforming meshes can be coupled with other numerical or quasi-analytical techniques, such as normal-mode summation [Capdeville et al., 2003], but this is beyond the scope of this article.

The shape of the physical grid is formulated by a mapping (deformation) of a reference cube. Cartesian points $\mathbf{x}=(x$, $y, z$ ) within a given deformed, hexahedral element $\Omega_{e}$ are mapped to the reference cube based upon the transform 

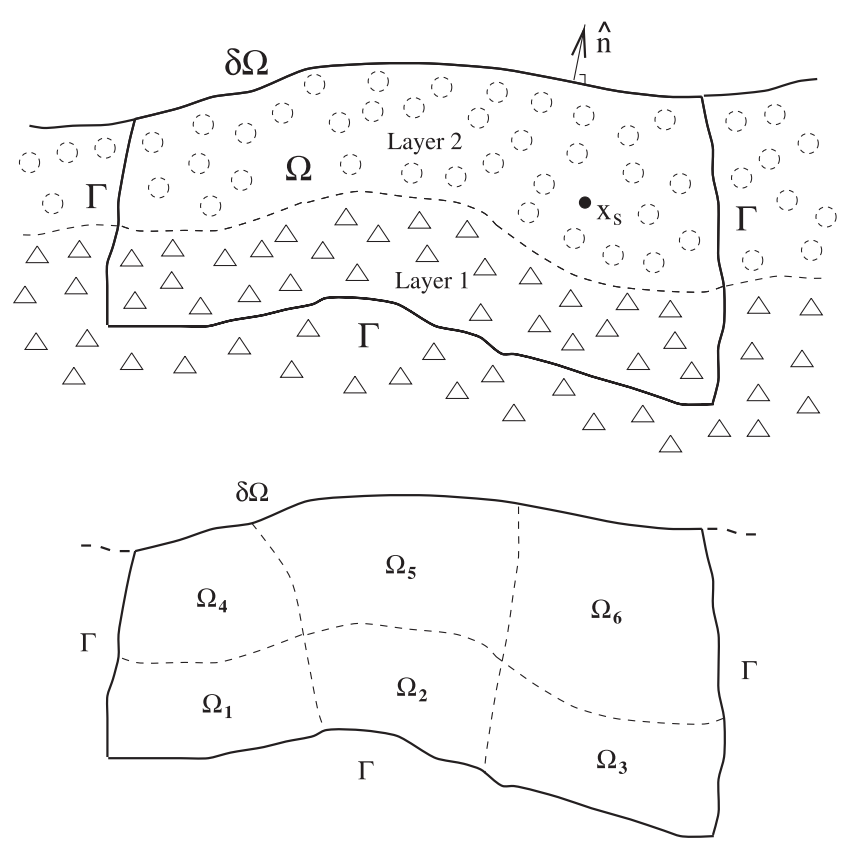

Figure 1. Finite Earth model with volume $\Omega$ and free surface $\partial \Omega$ (top). $\Gamma$ is the artificial absorbing boundary, and $\hat{\mathbf{n}}$ the unit outward normal on the surface. $\mathbf{x}_{\mathbf{s}}$ indicates the location of the source. The model is subdivided into curved spectral elements using quadrangles in 2-D and hexahedra in 3-D (bottom). The shape of the elements is adapted to all the major discontinuities in the geological model, i.e., surface topography, main layers, and faults. Inside each element, the model can be heterogeneous.

$$
\mathbf{x}(\boldsymbol{\xi})=\sum_{a=1}^{n_{a}} N_{a}(\boldsymbol{\xi}) \mathbf{x}_{a}
$$

Points within the reference cube are denoted by the vector $\xi=(\xi, \eta, \zeta)$, where $-1 \leq \xi \leq 1,-1 \leq \eta \leq 1$ and $-1 \leq \zeta \leq 1$. In our implementation, the geometry of the spectral elements is controlled by $n_{a}=27$ points, or anchors, $\mathbf{x}_{a}$, as shown in Figure 2. The $n_{a}$ shape functions $N_{a}$ are triple products of 1-D degree-2 Lagrange polynomials in the three orthogonal directions of space in the reference cube. The three Lagrange polynomials of degree 2 with three control points $\xi_{0}=-1$, $\xi_{1}=0$, and $\xi_{2}=1$ are $\ell_{0}^{2}(\xi)=\frac{1}{2} \xi(\xi-1), \ell_{1}^{2}(\xi)=1-\xi^{2}$, and $\ell_{2}^{2}(\xi)=\frac{1}{2} \xi(\xi+1)$.

The Jacobian $J$ of the mapping $J=|\partial(x, y, z) / \partial(\xi, \eta, \zeta)|$ is used to define the relationship between a small volume $\mathrm{d} x \mathrm{~d} y \mathrm{~d} z$ within a given finite element and a volume $\mathrm{d} \xi \mathrm{d} \eta$ $\mathrm{d} \zeta$ in the reference cube:

$$
\mathrm{d} x \mathrm{~d} y \mathrm{~d} z=J \mathrm{~d} \xi \mathrm{d} \eta \mathrm{d} \zeta .
$$

The partial derivative matrix $\partial \mathbf{x} / \partial \xi$ needed for the calculation of the Jacobian is obtained by analytically differentiating

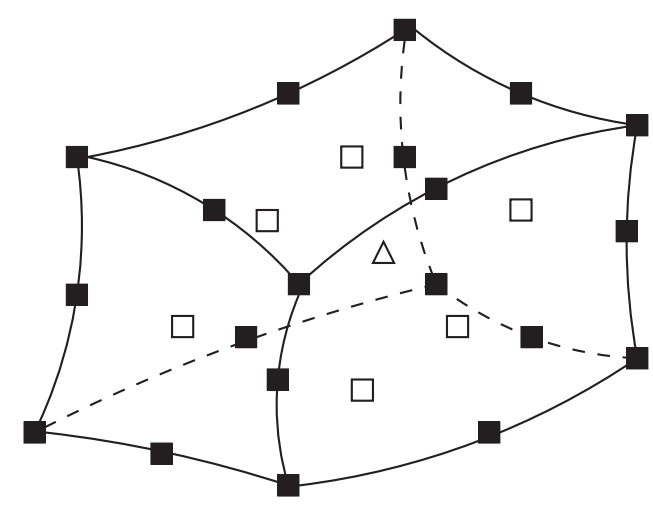

Figure 2. The geometry of each of the curved hexahedra is defined by 27 control nodes, or anchors. Lagrange interpolants of degree 2 at these control points allow one to compute the Jacobian matrix of the transformation between the reference cube and the deformed spectral element.

the mapping (1). The partial derivatives of the shape functions $N_{a}$ are expressed in terms of Lagrange polynomials of degree 2 and their derivatives at the 27 control points.

As in any FEM [e.g., Hughes, 1987], the Jacobian matrix plays a critical role in designing a good mesh for a realistic 3-D structure. First, the determinant of the Jacobian matrix $J$ should never vanish to ensure that the local mapping (1) is unique and invertible. Second, local variations of the Jacobian should be smooth everywhere within the mesh. Sharp local variations indicate highly-distorted elements that lead to inaccurate or even unstable calculations.

For the implementation of absorbing boundary conditions as well as in the context of fluid-solid coupling, surface integrals need to be evaluated. Because the mesh of hexahedra $\Omega_{e}$ honors the major discontinuities in the model, the surfaces are naturally divided in terms of non-overlapping quadrilateral surface elements $\Gamma_{b}$ that are isomorphous to the square. For any given boundary element, the relation between a point $\mathbf{x}$ within the element and a point $(\xi, \eta)$ in the reference square may be written in terms of 2-D shape functions $N_{a}(\xi, \eta)$ and anchors $\mathbf{x}_{a}$ in the form

$$
\mathbf{x}(\xi, \eta)=\sum_{a=1}^{n_{a}} N_{a}(\xi, \eta) \mathbf{x}_{a}
$$

In this case the shape functions are products of 1-D degree2 Lagrange polynomials. Given this mapping, the normal $\hat{\mathbf{n}}$ to a boundary element $\Gamma_{b}$ is given by

$$
\hat{\mathbf{n}}=\frac{1}{J_{b}} \frac{\partial \mathbf{x}}{\partial \xi} \times \frac{\partial \mathbf{x}}{\partial \eta}
$$


where $J_{b}$ denotes the Jacobian of the transformation:

$$
J_{b}=\left\|\frac{\partial \mathbf{x}}{\partial \xi} \times \frac{\partial \mathbf{x}}{\partial \eta}\right\| .
$$

The relationship between a small surface element $\mathrm{d} x \mathrm{~d} y$ and a surface $\mathrm{d} \xi \mathrm{d} \eta$ in the reference square is then

$$
\mathrm{d} x \mathrm{~d} y=J_{b} \mathrm{~d} \xi \mathrm{d} \eta \text {. }
$$

To avoid a staircase discretization of major interfaces and the related spurious diffraction that appears in methods based on a regular grid of points, a good mesh should honor all the major discontinuities in the model (Figure 1). In addition, wave speed usually increases with depth (e.g., sediments above bedrock at the local or regional scale, or crust above mantle on a global scale), and ideally one wants to increase the size of the elements with depth in order to maintain a similar number of points per wavelength everywhere in the model (i.e., to provide the same numerical resolution everywhere).

To illustrate how to construct a mesh in practice for the globe, we explain how to build a grid designed to match the 1-D Preliminary Reference Earth Model (PREM) [Dziewonski and Anderson, 1981]. Following the ideas of Taylor et al. [1997] and Chaljub [2000], we first decompose the sphere into six blocks using the concept of the 'quasi-uniform gnomonic projection', or 'cubed sphere' [Sadourny, 1972; Ronchi et al., 1996], as illustrated in Figure 3. We then mesh each of the six blocks, making sure that they match perfectly at their common interfaces. Following Chal$j u b$ [2000], the singularity of coordinates at the Earth's center

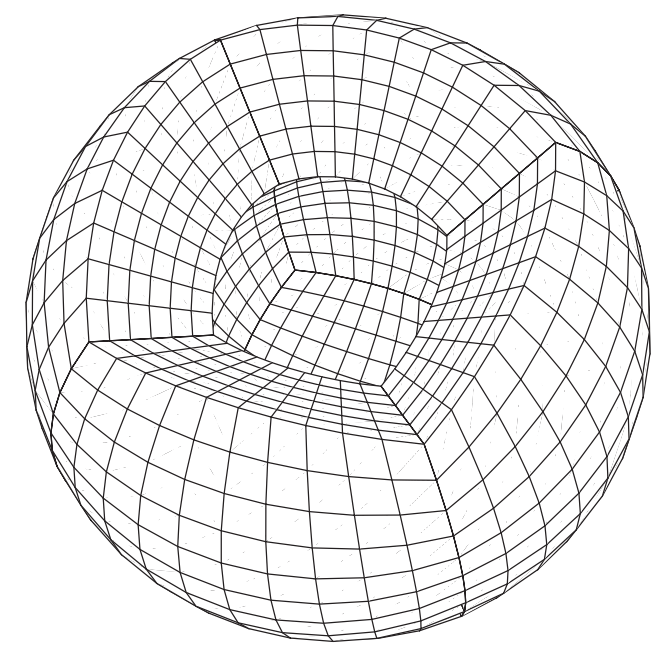

Figure 3. The 'cubed sphere' decomposition of the spherical Earth into six blocks. One of the six blocks has been removed for clarity. is avoided by placing a small cube around the center of the inner core. The mesh within this cube matches up with the cubed sphere mesh at the inner-core boundary (ICB). As mentioned above, our final mesh needs to honor all firstorder discontinuities in PREM, which are the middle crust at a depth of $15 \mathrm{~km}$, the Moho at a depth of $24.4 \mathrm{~km}$, the upper mantle discontinuities at depths of $220 \mathrm{~km}, 400 \mathrm{~km}$, and $670 \mathrm{~km}$, the core-mantle boundary (CMB), and the ICB; it also honors second-order discontinuities at $600 \mathrm{~km}$, $771 \mathrm{~km}$, and at the top of D'. The density of the mesh is increased in the upper part of the model (crust and upper mantle) based upon a set of geometrical doubling mesh cells (Figure 4). A first doubling region is introduced below the Moho, a second below the $670 \mathrm{~km}$ discontinuity, and a third just above the ICB. Note that for other classical models, such as IASP91 [Kennett and Engdahl, 1991], the mesh would be slightly modified because it would need to honor a set of major discontinuities located at slightly different depths.

For our 3-D global mesh we use mantle model S20RTS of Ritsema et al. [1999], whose lateral variations are superimposed on PREM. Variations in density are obtained by scaling the shear-wave speed variations by a factor of 0.4 , in accordance with mineral physics estimates [Anderson, 1987; Karato, 1993]. For the crust we use model Crust 2.0 [Bassin et al., 2000], which is a global $2^{\circ} \times 2^{\circ}$ model. We implement a smooth, interpolated version of this crustal model to define the compressional- and shear-wave speeds at existing grid points in our mesh. We do not adapt our mesh to the shape of the Moho or intracrustal discontinuities given by this crustal model because we would need to signifi-

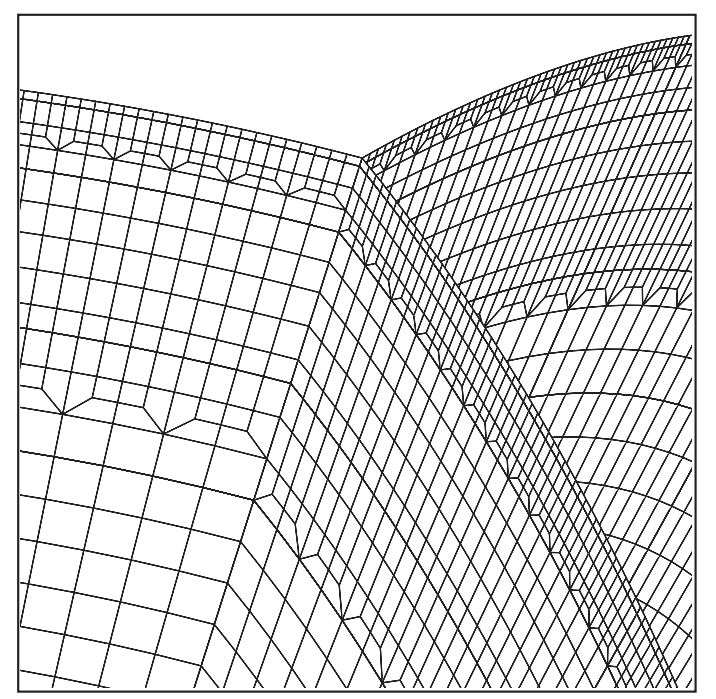

Figure 4. Close-up of the two geometrical mesh doubling regions in the mantle. 
cantly increase the number of grid elements in the crust and right below the Moho, which in turn would significantly increase the cost of the numerical simulations. Presently, the shape of these intracrustal interfaces and the Moho is insufficiently known to warrant this.

Once the mantle and crustal models have been added, we make the Earth elliptical in shape. Our mesh incorporates a smoothed version of global topography and bathymetry. The bathymetry map is also used to define the thickness of the oceans at the surface of the mesh in order to take into account the effects of the oceans on global wave propagation, as will be explained in Section 3.5.

The quality of the mesh can be expressed in terms of the number of grid points per wavelength, i.e., the resolution of the mesh in terms of how well it samples the wave field, $N=\tau_{0}(v / \Delta h)_{\min }$. Here $\tau_{0}$ denotes the shortest period of the source and $(v / \Delta h)_{\min }$ denotes the minimum ratio of shearwave or surface-wave speed $v$ and grid spacing $\Delta h$ within a given spectral element in the mesh. Because surface waves are slower than shear waves, in elements located at the free surface it is the surface-wave speed that controls the resolution of the mesh, not the shear-wave speed; inside the model it is the shear-wave speed that matters.

\section{SOLVING THE WAVE EQUATION ON A SPECTRAL-ELEMENT GRID}

\subsection{The Weak Form of the Seismic Wave Equation}

The differential form of the seismic wave equation is classically written in the form

$$
\rho \partial_{t}^{2} \mathbf{s}=\boldsymbol{\nabla} \cdot \mathbf{T}+\mathbf{f},
$$

where $\rho$ denotes the 3-D distribution of density and $\mathbf{T}$ the stress tensor, which is linearly related to the displacement gradient $\nabla \mathbf{s}$ by Hooke's law:

$$
\mathbf{T}=\mathbf{c}: \boldsymbol{\nabla} \mathbf{s} .
$$

We make no particular assumption on the structure of the stiffness tensor $\mathbf{c}$ that describes the properties of the medium, i.e., the formulation is general and can handle a fully anisotropic tensor with 21 independent coefficients. In an attenuating medium, the stress is determined by the entire strain history, and Hooke's law (8) becomes:

$$
\mathbf{T}(t)=\int_{-\infty}^{t} \partial_{t} \mathbf{c}\left(t-t^{\prime}\right): \nabla \mathbf{s}\left(t^{\prime}\right) \mathrm{d} t^{\prime} .
$$

In seismology, the quality factor $Q$ is generally observed to be approximately constant over a wide range of frequencies.
To approximate such an absorption-band solid, Liu et al. [1976] introduced the idea of using a series of $L$ standard linear solids [e.g., Emmerich and Korn, 1987; Carcione et al., 1988; Moczo et al., 1997]. An almost constant $Q$ can usually be approximated with a reasonable level of accuracy using three such linear solids. In the Earth, the bulk quality factor is several hundred times larger than the shear quality factor, which means that attenuation mainly depends on the shear quality factor. Therefore as far as attenuation is concerned one can safely assume that it is sufficient to model the time evolution of the average isotropic shear modulus. As a consequence, following Liu et al. [1976], we write:

$$
\mu(t)=\mu_{R}\left[1-\sum_{\ell=1}^{L}\left(1-\tau_{\ell}^{\epsilon} / \tau_{\ell}^{\sigma}\right) e^{-t / \tau_{\ell}^{\sigma}}\right] H(t) .
$$

Here $\mu_{R}$ denotes the relaxed modulus, $H(t)$ is the Heaviside function, and $\tau_{\ell}^{\sigma}$ and $\tau_{\ell}^{\epsilon}$ denote the stress and strain relaxation times, respectively, of the $\ell$-th standard linear solid. Using the absorption-band shear modulus (10), the constitutive relation (9) becomes

$$
\mathbf{T}=\mathbf{c}_{U}: \nabla \mathbf{s}-\sum_{\ell=1}^{L} \mathrm{R}_{\ell}
$$

where $\mathbf{c}_{U}$ is the unrelaxed elastic tensor determined by the unrelaxed shear modulus

$$
\mu_{U}=\mu_{R}\left[1-\sum_{\ell=1}^{L}\left(1-\tau_{\ell}^{\epsilon} / \tau_{\ell}^{\sigma}\right]\right.
$$

For each standard linear solid we therefore have to solve the so-called 'memory variable' equation

$$
\partial_{t} \mathbf{R}_{\ell}=-\left(\mathbf{R}_{\ell}-\delta \mu_{\ell} \mathbf{D}\right) / \tau_{\ell}^{\sigma},
$$

where $\mathbf{D}$ is the strain deviator:

$$
\mathbf{D}=\frac{1}{2}\left[\boldsymbol{\nabla} \mathbf{s}+(\boldsymbol{\nabla} \mathbf{s})^{T}\right]-\frac{1}{3}(\boldsymbol{\nabla} \cdot \mathbf{s}) \mathbf{I} .
$$

Here a superscript $T$ denotes the transpose and $\mathbf{I}$ is the identity tensor. The memory-variable tensors $\mathbf{R}_{\ell}$ are symmetric and have zero trace, such that each standard linear solid introduces five additional unknowns. The modulus defect $\delta \mu_{\ell}$ associated with each individual standard linear solid is determined by

$$
\delta \mu_{\ell}=-\mu_{R}\left(1-\tau_{\ell}^{\epsilon} / \tau_{\ell}^{\sigma}\right) .
$$


If the earthquake can be represented by a point source, the force $\mathbf{f}$ in (7) may be written in terms of the moment tensor $\mathbf{M}$ as [Dahlen and Tromp, 1998]:

$$
\mathbf{f}=-\mathbf{M} \cdot \nabla \delta\left(\mathbf{x}-\mathbf{x}_{\mathrm{s}}\right) S(t) .
$$

The location of the point source is denoted by $\mathbf{x}_{\mathrm{s}}, \delta\left(\mathbf{x}-\mathbf{x}_{\mathrm{s}}\right)$ is the Dirac delta distribution located at $\mathbf{x}_{\mathrm{s}}$, and $S(t)$ is the source-time function. In the case of a source of finite size, such as a fault plane $\sum_{\mathrm{s}}$, the source term can be written in terms of the moment-density tensor $\mathbf{m}$ as

$$
\mathbf{f}=-\mathbf{m}\left(\mathbf{x}_{\mathrm{s}}, t\right) \cdot \nabla \delta\left(\mathbf{x}-\mathbf{x}_{\mathrm{s}}\right) \text { on } \Sigma_{s}
$$

In what follows we will use the finite source (17) for reasons of generality.

As illustrated in Figure 1, two types of boundary conditions must be considered: on the free surface $\partial \Omega$ the traction $\hat{\mathbf{n}}$ - T, where $\hat{\mathbf{n}}$ denotes the unit outward normal on the free surface, vanishes, and in the case of local or regional simulations, seismic energy needs to be absorbed on the fictitious boundaries $\Gamma$ of the domain, in order to mimic a semi-infinite medium. To accomplish the latter, one usually uses a paraxial equation to damp the wave field on the edges [Clayton and Engquist, 1977; Quarteroni et al., 1998], for instance

$$
\begin{aligned}
\mathbf{T} \cdot \hat{\mathbf{n}} & =\boldsymbol{\rho}\left[v_{n}\left(\hat{\mathbf{n}} \cdot \partial_{t} \mathbf{s}\right) \hat{\mathbf{n}}\right. \\
& \left.+v_{1}\left(\hat{\mathbf{t}}_{1} \cdot \partial_{t} \mathbf{s}\right) \hat{\mathbf{t}}_{1}+v_{2}\left(\hat{\mathbf{t}}_{2} \cdot \partial_{t} \mathbf{s}\right) \hat{\mathbf{t}}_{2}\right],
\end{aligned}
$$

where $\hat{\mathbf{t}}_{1}$ and $\hat{\mathbf{t}}_{2}$ are orthogonal unit vectors tangential to the absorbing boundary $\Gamma$ with unit outward normal $\hat{\mathbf{n}}, v_{n}$ is the quasi-P wave speed of waves traveling in the $\hat{\mathbf{n}}$ direction, $v_{1}$ is the quasi-S wave speed of waves polarized in the $\hat{\mathbf{t}}_{1}$ direction, and $v_{2}$ is the quasi-S wave speed of waves polarized in the $\hat{\mathbf{t}}_{2}$ direction. The absorbing boundary condition (18) perfectly absorbs waves impinging at a right angle to the boundary, but is less effective for waves that graze the boundary [Clayton and Engquist, 1977]. It is valid for transversely isotropic media with a horizontal or vertical symmetry axis; general anisotropy can be accommodated by tapering it such that the medium becomes transversely isotropic on the absorbing boundary $\Gamma$. It is worth mentioning that in recent years a significantly more efficient absorbing condition called the Perfectly Matched Layer (PML) has been introduced [Bérenger, 1994; Collino and Tsogka, 2001], and it has been shown recently that it can be adapted to SEMs [Komatitsch and Tromp, 2003; Basu and Chopra, 2004; Festa and Vilotte, 2005]. In the near future, this condition could replace classical paraxial equations such as that of Clayton and Engquist [1977] in existing FEM or SEM codes.
The differential form of the equation of motion (7), which is frequently called the 'strong' formulation of the problem, is used in many classical numerical techniques, such as finite-difference and pseudospectral methods. In FEMs or SEMs, however, one works with a modified version of the equation called the integral or 'weak' formulation of the problem. It is obtained by first taking the dot product of the momentum equation (7) with an arbitrary vector $\mathbf{w}$, which is called a test vector in the context of finite-element analysis. Next, one performs an integration by parts over the volume $\Omega$ of the model, imposing the boundary conditions mentioned above, which gives:

$$
\begin{aligned}
& \int_{\Omega} \rho \mathbf{w} \cdot \partial_{t}^{2} \mathbf{s} \mathrm{d}^{3} \mathbf{x}=-\int_{\Omega} \nabla \mathbf{w}: \mathbf{T} \mathrm{d}^{3} \mathbf{x} \\
& +\int_{\Sigma s} \mathbf{m}\left(\mathbf{x}_{\mathrm{s}}, t\right): \nabla \mathbf{w}\left(\mathbf{x}_{\mathrm{s}}\right) \mathrm{d}^{2} \mathbf{x}_{\mathrm{s}}+\int_{\Gamma} \hat{\mathbf{n}} \cdot \mathbf{T} \cdot \mathbf{w} \mathrm{d}^{2} \mathbf{x}
\end{aligned}
$$

Equation (19) is equivalent to the strong formulation (7) because it holds for any test vector w. Note that the source term (17) has been integrated explicitly using the properties of the Dirac delta distribution. Equation (19) illustrates why the SEM is very accurate for modeling surface waves: the traction-free surface condition is imposed naturally and automatically during the integration by parts, because the contour integral over the free surface $\partial \Omega$ in (19) simply vanishes. In other words, the free-surface condition is a natural condition of the problem. In the context of local and regional simulations, the last integral on the right-hand side of (19) involves the absorbing boundary $\Gamma$, which may be implemented based upon the one-way treatment (18). One of the nice aspects of simulating global wave propagation from a numerical point of view is that there are no absorbing boundaries, which simplifies the problem.

At long periods one needs to incorporate the effects of self gravitation and rotation on seismic wave propagation, which are mostly relevant for long-period surface waves. Such effects have been included in the SEM and lead to additional terms in the weak formulation (19). These terms were introduced in Chaljub [2000], Komatitsch and Tromp [2002b], Chaljub et al. [2003] and Chaljub and Valette [2004], and the corresponding effects on seismic waves were carefully benchmarked. We summarize these results in the following sections for completeness.

\subsection{The Wave Equation in the Mantle and the Crust}

In a rotating, self-gravitating Earth model, the elastic wave equation for the mantle and crust may be written in the form [Dahlen and Tromp, 1998] 


$$
\begin{aligned}
\rho\left(\partial_{t}^{2} \mathbf{s}+2 \mathbf{\Omega} \times \partial_{t} \mathbf{s}\right) & =\nabla \cdot \mathbf{T}+\nabla(\rho \mathbf{s} \cdot \mathrm{g})-\rho \nabla \boldsymbol{\phi} \\
& -\nabla \cdot(\rho \mathbf{s}) \mathbf{g}+\mathbf{f} .
\end{aligned}
$$

Here $\boldsymbol{\Omega}$ denotes the Earth's angular rotation vector, $\mathbf{g}$ the gradient of the geopotential, and $\mathbf{T}$ the stress tensor, which is linearly related to the displacement gradient $\nabla \mathbf{s}$ by Hooke's law (8) in an elastic model, or by the generalization (9) in an anelastic model. The earthquake source is represented by the force $\mathbf{f}$, which is given in terms of the momentdensity tensor $\mathbf{m}$ by (17). The perturbed gravitational potential $\phi$ is determined by Poisson's equation within the Earth, $\nabla^{2} \phi=-4 \pi G \nabla \cdot(\rho \mathbf{s})$, and by Laplace's equation in the rest of space, $\nabla^{2} \phi=0$.

Because Laplace's equation is defined in all of space, solving the momentum equation (20) in conjunction with Poisson's and Laplace's equations is difficult numerically. The approach can be simplified considerably by making what is known as Cowling's approximation [Cowling, 1941], as discussed by Valette [1987], Dahlen and Tromp [1998] and Chaljub et al. [2003]. In this approximation one ignores perturbations $\phi$ in the gravitational potential while retaining the unperturbed gravitational potential. Physically, this means that we ignore the effects of mass redistribution. Under this assumption the momentum equation (20) becomes

$\rho\left(\partial_{t}^{2} \mathbf{s}+2 \boldsymbol{\Omega} \times \partial_{t} \mathbf{s}\right)=\nabla \cdot \mathbf{T}+\nabla(\rho \mathbf{s} \cdot \mathbf{g})-\nabla \cdot(\rho \mathbf{s}) \mathbf{g}+\mathbf{f}$.

The associated boundary conditions are that on the free surface the traction $\hat{\mathbf{n}} \cdot \mathbf{T}$, where $\hat{\mathbf{n}}$ denotes the unit outward normal to the free surface, needs to vanish. On the CMB the normal component of displacement $\hat{\mathbf{n}} \cdot \mathbf{s}$ needs to be continuous, and the traction $\hat{\mathbf{n}} \cdot \mathbf{T}$ at the bottom of the mantle needs to match the traction $-p \hat{\mathbf{n}}$ at the top of the outer core, where $p$ denotes the perturbed pressure in the fluid.

The weak form of the equation of motion (21) is obtained by taking the dot product with an arbitrary test vector $\mathbf{w}$, integrating by parts over the volume $\mathrm{M}$ of the mantle and crust, and imposing the stress-free surface boundary condition. This gives

$$
\begin{aligned}
& \int_{M} \rho \mathbf{w} \cdot \partial_{t}^{2} \mathbf{s} \mathrm{d}^{3} \mathbf{x}+\int_{M} 2 \rho \mathbf{w} \cdot\left(\boldsymbol{\Omega} \times \partial_{t} \mathbf{s}\right) \mathrm{d}^{3} \mathbf{x}= \\
& -\int_{M} \nabla \mathbf{w}:(\mathbf{T}+\mathbf{G}) \mathrm{d}^{3} \mathbf{x}+\int_{\Sigma s} \mathbf{m}\left(\mathbf{x}_{\mathrm{s}}, t\right): \nabla \mathbf{w}\left(\mathbf{x}_{\mathrm{s}}\right) \mathrm{d}^{2} \mathbf{x}_{\mathrm{s}} \\
& -\int_{M} \rho \mathbf{s} \cdot \mathbf{H} \cdot \mathbf{w} \mathrm{d}^{3} \mathbf{x}+\int_{C M B} p \hat{\mathbf{n}} \cdot \mathbf{w} \mathrm{d}^{2} \mathbf{x},
\end{aligned}
$$

where we have used the continuity of traction at the CMB, and where we have defined the second-order tensors

$$
\mathbf{G}=\rho[\mathbf{s g}-(\mathbf{s} \cdot \mathbf{g}) \mathbf{I}]
$$

and

$$
\mathbf{H}=\nabla \mathbf{g}
$$

Because $\mathbf{G}$ is non-symmetric, let us note that our definition of the double dot product between two second-order tensors $\mathbf{A}$ and $\mathbf{B}$ is $\mathbf{A}: \mathbf{B}=A_{i j} B_{i j}$. The gravitational acceleration $\mathbf{g}$ is the gradient of a potential, and thus $\mathbf{H}$ is a symmetric second-order tensor.

\subsection{The Wave Equation in the Outer Core}

In the fluid outer core the equation of motion may be written in the form

$$
\begin{aligned}
\rho\left(\partial_{t}^{2} \mathbf{s}+2 \mathbf{\Omega} \times \partial_{t} \mathbf{s}\right) & =\nabla(\kappa \nabla \cdot \mathbf{s}+\rho \mathbf{s} \cdot \mathbf{g})-\rho \nabla \phi \\
& -\nabla \cdot(\rho \mathbf{s}) \mathbf{g},
\end{aligned}
$$

where $\kappa$ denotes the bulk modulus of the fluid. Under the assumption of hydrostatic equilibrium prior to the earthquake, the equation of motion in the fluid may be rewritten in the form

$$
\begin{aligned}
\partial_{t}^{2} \mathbf{s}+2 \boldsymbol{\Omega} \times \partial_{t} \mathbf{s} & =\nabla\left(\rho^{-1} \kappa \nabla \cdot \mathbf{s}+\mathbf{s} \cdot \mathbf{g}-\phi\right) \\
& +\rho^{-1} g^{-2} \kappa(\nabla \cdot \mathbf{s}) N^{2} \mathbf{g},
\end{aligned}
$$

where $g=|\mathbf{g}|$ and

$$
N^{2}=\left(\rho^{-1} \nabla \rho-\rho \kappa^{-1} \mathbf{g}\right) \cdot \mathbf{g}
$$

is the Brunt-Väisälä frequency [e.g., Valette, 1986; Dahlen and Tromp, 1998; Chaljub et al., 2003; Chaljub and Valette, 2004].

In previous work [Komatitsch and Tromp, 2002b] we made the assumption that the outer core was stably stratified and isentropic, which meant that $N^{2}=0$, and that perturbations in gravity could be ignored, i.e., $\phi=0$, based upon the Cowling approximation. Chaljub and Valette [2004] recently introduced a formulation of self-gravitation in a fluid medium that is valid for $N^{2} \neq 0$ and that does not require the Cowling approximation. Following their work, and extending it by incorporating the effects of rotation, we will obtain a weak implementation that does not involve these approximations. This improved formulation is important in practice because compared to the implementation in Komatitsch and Tromp [2002b] it does not require numerical iterations on the fluid-solid coupling condition at the CMB or at the ICB [Chaljub and Valette, 2004], thus making the 
SEM algorithm much simpler and more efficient, as will be illustrated in Section 4.

We express the displacement field $\mathbf{s}$ in terms of a scalar potential $\chi$ and a vector $\mathbf{u}$ as

$$
\mathbf{s}=\nabla \chi+\mathbf{u}
$$

where $\chi$ and $\mathbf{u}$ remain to be determined. Note that in Komatitsch and Tromp [2002b] we expressed velocity $\partial_{t} \mathbf{s}$ in terms of another potential $\chi$ and a vector $\mathbf{u}$ instead. Upon substitution of the representation (28) in (26) we obtain

$$
\begin{aligned}
& \nabla \partial_{t}^{2} \chi+\partial_{t}^{2} \mathbf{u}+2 \boldsymbol{\Omega} \times \nabla \partial_{t} \chi+2 \boldsymbol{\Omega} \times \partial_{t} \mathbf{u}= \\
& \quad \nabla\left(\rho^{-1} \kappa \nabla^{2} \chi+\rho^{-1} \kappa \nabla \cdot \mathbf{u}+\mathbf{g} \cdot \nabla \chi+\mathbf{g} \cdot \mathbf{u}-\phi\right) \\
& \quad+\rho^{-1} g^{-2} \kappa\left(\nabla^{2} \chi+\nabla \cdot \mathbf{u}\right) N^{2} \mathbf{g} .
\end{aligned}
$$

Let $\chi$ be determined by

$$
\partial_{t}^{2} \chi=\rho^{-1} \kappa \nabla \cdot(\nabla \chi+\mathbf{u})+\mathbf{g} \cdot(\nabla \chi+\mathbf{u})-\phi,
$$

then $\mathbf{u}$ satisfies

$$
\begin{aligned}
& \partial_{t}^{2} \mathbf{u}+2 \boldsymbol{\Omega} \times \partial_{t} \mathbf{u}=-2 \boldsymbol{\Omega} \times \nabla \partial_{t} \chi \\
& \quad+g^{-2}\left[\partial_{t}^{2} \chi-\mathbf{g} \cdot(\nabla \chi+\mathbf{u})+\phi\right] N^{2} \mathbf{g}
\end{aligned}
$$

At the ICB and CMB we need to exchange pressure $p$ between the fluid and the solid. Using (28) and (30) we have

$$
\begin{gathered}
p=-\kappa \nabla \cdot \mathbf{s}=-\rho\left[\partial_{t}^{2} \chi\right. \\
-\mathbf{g} \cdot(\nabla \chi+\mathbf{u})+\phi] .
\end{gathered}
$$

The weak form of (30) is obtained by multiplying it by an arbitrary test function $w$ and integrating by parts, using the continuity of the normal component of displacement:

$$
\begin{aligned}
\int_{O C} & \kappa^{-1} \rho w \partial_{t}^{2} \chi d^{3} \mathbf{x}=-\int_{O C}(\nabla w) \cdot(\nabla \chi+\mathbf{u}) d^{3} \mathbf{x} \\
& +\int_{O C} \kappa^{-1} \rho w[\mathbf{g} \cdot(\nabla \chi+\mathbf{u})-\phi] d^{3} \mathbf{x} \\
& +\int_{C M B} w \hat{\mathbf{n}} \cdot \mathbf{s} d^{2} \mathbf{x}-\int_{I C B} w \hat{\mathbf{n}} \cdot \mathbf{s} d^{2} \mathbf{x} .
\end{aligned}
$$

The weak form of (31) is obtained by dotting it with a test vector w:

$$
\begin{aligned}
\int_{O C} \mathbf{w} \cdot \partial_{t}^{2} \mathbf{u} d^{3} \mathbf{x}= \\
-2 \int_{O C} \mathbf{w} \cdot\left[\mathbf{\Omega} \times\left(\partial_{t} \mathbf{u}+\nabla \partial_{t} \chi\right)\right] d^{3} \mathbf{x} \\
-\int_{O C} \rho^{-1} g^{-2} p N^{2} \mathbf{w} \cdot \mathbf{g} d^{3} \mathbf{x}
\end{aligned}
$$

where we have used (32). Making the Cowling approximation involves simply setting $\phi$ equal to zero in (30)-(34). If we make the Cowling approximation and set the BruntVäisälä frequency $N$ equal to zero, as in Komatitsch and Tromp [2002b], we obtain the following equation for $\chi$ :

$$
\partial_{t}^{2} \chi=\rho^{-1} \kappa \nabla \cdot(\nabla \chi+\mathbf{u})+\mathbf{g} \cdot(\nabla \chi+\mathbf{u}),
$$

and for $\mathbf{u}$ :

$$
\partial_{t} \mathbf{u}+2 \boldsymbol{\Omega} \times \mathbf{u}=-2 \boldsymbol{\Omega} \times \nabla_{\chi}
$$

The pressure $p$ in (32) becomes

$$
p=-\rho\left[\partial_{t}^{2} \chi-\mathbf{g} \cdot(\nabla \chi+\mathbf{u})\right] .
$$

In the absence of rotation (31) reduces to the scalar equation

$$
\partial_{t}^{2} u+N^{2} u=g^{-1}\left[\partial_{t}^{2} \chi-\mathbf{g} \cdot \nabla \chi+\phi\right] N^{2},
$$

where $u$ is determined by $\mathbf{u}=u \mathbf{g} / \mathrm{g}$. Assuming further that the Brunt-Väisälä frequency $N$ equals zero implies $\mathbf{u}=\mathbf{0}$ and

$$
\partial_{t}^{2} \chi=\rho^{-1} \kappa \nabla^{2} \chi+\mathbf{g} \cdot \nabla \chi-\phi
$$

Under these assumptions the pressure reduces to

$$
p=-\rho\left(\partial_{t}^{2} \chi-\mathbf{g} \cdot \nabla \chi+\phi\right)
$$

Finally, if we also ignore the effects of self-gravitation we obtain

$$
\partial_{t}^{2} \chi=\rho^{-1} \kappa \nabla^{2} \chi
$$

and the pressure becomes

$$
p=-\rho \partial_{t}^{2} \chi
$$

This final approximation amounts to solving the acoustic wave equation in the outer core.

In the domain decomposition between the fluid outer core and the solid inner core and mantle, we match the normal component of displacement by taking $\hat{\mathbf{n}} \cdot \mathbf{s}$ at the bottom of the mantle and using it in the surface integral over the CMB in (33), and by taking $\hat{\mathbf{n}} \cdot \mathbf{s}$ from the top of the inner core and using it in the surface integral over the ICB in (33). The continuity of traction is honored by calculating the fluid pressure $p$ from (37) based upon $\partial_{t}^{2} \chi$ in the fluid and the normal component of displacement $\hat{\mathbf{n}} \cdot \mathbf{s}=\hat{\mathbf{n}} \cdot(\nabla \chi+\mathbf{u})$ taken from the solid at the bottom of the mantle (CMB) or at the top of the inner core (ICB). 


\subsection{The Wave Equation in the Inner Core}

The weak form of the equation of motion in the solid inner core is similar to (22):

$$
\begin{aligned}
\int_{I C} \rho \mathbf{w} \cdot \partial_{t}^{2} \mathbf{s} \mathrm{d}^{3} \mathbf{x}+\int_{I C} 2 \rho \mathbf{w} \cdot\left(\boldsymbol{\Omega} \times \partial_{t} \mathbf{s}\right) \mathrm{d}^{3} \mathbf{x}= \\
\quad-\int_{I C} \nabla \mathbf{w}:(\mathbf{T}+\mathbf{G}) \mathrm{d}^{3} \mathbf{x} \\
\quad-\int_{I C} \rho \mathbf{s} \cdot \mathbf{H} \cdot \mathbf{w} \mathrm{d}^{3} \mathbf{x}-\int_{I C B} p \hat{\mathbf{n}} \cdot \mathbf{w} \mathrm{d}^{2} \mathbf{x}
\end{aligned}
$$

Note that the inner core-outer core interactions represented by the surface integrals over the ICB in (33) and (43) also honor continuity in traction and continuity of the normal component of displacement and velocity.

\subsection{Additional Terms to Model the Effect of the Oceans}

We also seek to include the effect of the oceans, which is mostly relevant for free-surface reflected phases, such as PP, SS, and SP, and for the dispersion of Rayleigh waves. Those areas of the Earth that are covered by oceans are therefore subject to a slightly more complicated weak formulation of the problem. The weak form of the equations of motion in the solid Earth (mantle and crust) covered by water is

$$
\begin{aligned}
\int_{M} \rho \mathbf{w} & \cdot \partial_{t}^{2} \mathbf{s} \mathrm{d}^{3} \mathbf{x}+\int_{M} 2 \rho \mathbf{w} \cdot\left(\boldsymbol{\Omega} \times \partial_{t} \mathbf{s}\right) \mathrm{d}^{3} \mathbf{x}= \\
& -\int_{M} \nabla \mathbf{w}:(\mathbf{T}+\mathbf{G}) \mathrm{d}^{3} \mathbf{x} \\
& +\int_{\Sigma_{S}} \mathbf{m}\left(\mathbf{x}_{\mathrm{s}}, t\right): \nabla \mathbf{w}\left(\mathbf{x}_{\mathrm{s}}\right) \mathrm{d}^{2} \mathbf{x}_{\mathrm{s}} \\
& -\int_{M} \rho \mathbf{s} \cdot \mathbf{H} \cdot \mathbf{w} \mathrm{d}^{3} \mathbf{x}+\int_{C M B} p \hat{\mathbf{n}} \cdot \mathbf{w} \mathrm{d}^{2} \mathbf{x} \\
& -\int_{O C B} p \hat{\mathbf{n}} \cdot \mathbf{w} \mathrm{d}^{2} \mathbf{x},
\end{aligned}
$$

where OCB denotes the ocean-crust boundary (i.e., the ocean floor). We then need an expression for the fluid pressure $p$ at the OCB. In the oceans the waves satisfy the fluid wave equation (26), which, making the Cowling approximation and setting $N^{2}=0$, can be rewritten as:

$$
\partial_{t}^{2} \mathbf{s}+2 \mathbf{\Omega} \times \partial_{t} \mathbf{s}=-\nabla(p-\mathbf{s} \cdot \mathbf{g})
$$

In Komatitsch and Tromp [2002b], we made the assumption that the oceans were incompressible, which meant that the entire water column moved as a whole as a result of the normal displacement $\hat{\mathbf{n}} \cdot \mathbf{s}$ of the sea floor. We obtained the local expression for pressure at the ocean bottom

$p=\rho_{w} h \hat{\mathbf{n}} \cdot \partial_{t}^{2} \mathbf{s}+2 \rho_{w} h \hat{\mathbf{n}} \cdot\left(\boldsymbol{\Omega} \times \partial_{t} \mathbf{s}\right)+4 \pi G \rho_{w}^{2} h \hat{\mathbf{n}} \cdot \mathbf{s}$,

where $\rho_{w}$ denotes the density of sea water, and where the local thickness of the oceans, $h$, can be taken from a global bathymetry map. As a result, the weak form of the equation of motion in the crust and mantle (44) becomes

$$
\begin{aligned}
\int_{M} \rho \mathbf{w} \cdot \partial_{t}^{2} \mathbf{s} \mathrm{d}^{3} \mathbf{x}+\int_{M} 2 \rho \mathbf{w} \cdot\left(\boldsymbol{\Omega} \times \partial_{t} \mathbf{s}\right) \mathrm{d}^{3} \mathbf{x} \\
\quad+\int_{O C B} \rho_{w} h(\mathbf{w} \cdot \hat{\mathbf{n}})\left(\hat{\mathbf{n}} \cdot \partial_{t}^{2} \mathbf{s}\right) \mathrm{d}^{2} \mathbf{x}= \\
\quad-\int_{M} \nabla \mathbf{w}:(\mathbf{T}+\mathbf{G}) \mathrm{d}^{3} \mathbf{x} \\
\quad+\int_{\Sigma_{S}} \mathbf{m}\left(\mathbf{x}_{\mathrm{s}}, t\right): \nabla \mathbf{w}\left(\mathbf{x}_{\mathrm{s}}\right) \mathrm{d}^{2} \mathbf{x}_{\mathrm{s}} \\
\quad-\int_{M} \rho \mathbf{s} \cdot \mathbf{H} \cdot \mathbf{w} \mathrm{d}^{3} \mathbf{x}+\int_{C M B} p \hat{\mathbf{n}} \cdot \mathbf{w} \mathrm{d}^{2} \mathbf{x}
\end{aligned}
$$

This means that the effect of the oceans can be taken into account very efficiently by a simple modification of the mass matrix for the degrees of freedom located at the ocean floor. Unfortunately, this approximation becomes invalid at periods shorter than typically 20 s. Below this threshold, the thickness of the ocean layer cannot be neglected, and the fluid wave equation needs to be solved numerically in the oceans and coupled at the ocean bottom with the solid wave equation in the crust and the mantle. This is not an easy task, because the compressional-wave speed in the oceans is much smaller than that in the crust, and because the thickness of the oceans varies considerably, complicating mesh design.

\subsection{Definition of the Wave Field on an Element}

After meshing the model and expressing the seismic wave equation in its weak form, we need to define basis functions to represent the unknown displacement vector and the test vector on each element. In most FEMs, both the geometry of the problem and the vector fields are expressed using low-degree polynomials. In the SEM, the geometry of the curved elements is also usually defined using low-degree polynomials, as explained above, but all unknown functions are defined using higher-degree polynomials. This is a major difference between SEMs and FEMs. In this regard, SEMs are related to so-called $h-p$ FEMs, which also use polynomials of higher degree (but result in a non-diagonal mass ma- 
trix) [e.g., Guo and Babuška, 1986]. Typically, a SEM uses Lagrange polynomials of degree 4 to 10 for the interpolation of functions. The $n+1$ Lagrange polynomials of degree $n$ (Figure 5) are defined in terms of $n+1$ control points $-1 \leq$ $\xi_{\alpha} \leq 1, \alpha=0, \ldots, n$, by

$$
\begin{aligned}
& \ell_{\alpha}^{n}(\xi)= \\
& \quad \frac{\left(\xi-\xi_{0}\right) \ldots\left(\xi-\xi_{\alpha-1}\right)\left(\xi-\xi_{\alpha+1}\right) \ldots\left(\xi-\xi_{n}\right)}{\left(\xi_{\alpha}-\xi_{0}\right) \ldots\left(\xi_{\alpha}-\xi_{\alpha-1}\right)\left(\xi_{\alpha}-\xi_{\alpha+1}\right) \ldots\left(\xi_{\alpha}-\xi_{n}\right)} .
\end{aligned}
$$

As a result of this definition, the Lagrange polynomials are equal to either zero or one at any given control point:

$$
\ell_{\alpha}^{n}\left(\xi_{\beta}\right)=\delta_{\alpha \beta},
$$

where $\delta$ denotes the Kronecker delta. In a SEM, the control points $\xi_{\alpha, \alpha}=0, \ldots, n$, needed in the definition (48) are chosen to be the $n+1$ Gauss-Lobatto-Legendre (GLL) points (Figure 5), which are the roots of $\left(1-\xi^{2}\right) P_{n}^{\prime}(\xi)=0$, where $P_{n}^{\prime}$ denotes the derivative of the Legendre polynomial of degree $n$ [Canuto et al., 1988]. As will be explained later, this choice is motivated by the fact that the combination of Lagrange interpolants with GLL quadrature leads to an exactly diagonal mass matrix. As a result of this choice, each spectral element contains a grid of $(n+1)^{3}$ GLL points, and each edge of an element contains a grid of $(n+1)^{2}$ GLL points, as illustrated in Figure 6.

Functions $f$ on an element are interpolated in terms of triple products of Lagrange polynomials as

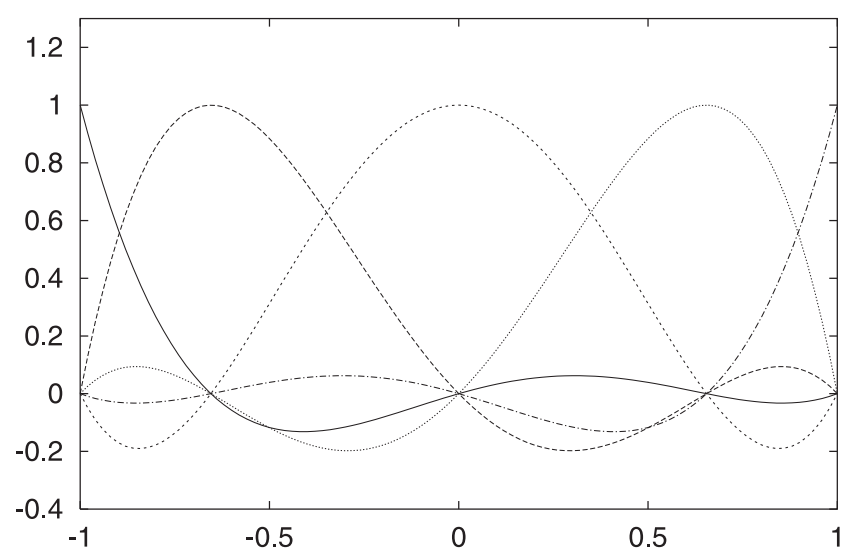

Figure 5. Lagrange interpolants of degree $N=4$ on the reference segment $[-1,1]$. The $N+1=5$ Gauss-Lobatto-Legendre points can be distinguished along the horizontal axis. All Lagrange polynomials are, by definition, equal to 1 or 0 at each of these points. Note that the first and last points are exactly -1 and 1 .

$$
\begin{gathered}
f(\mathbf{x}(\xi, \eta, \zeta)) \\
\approx \sum_{\alpha, \beta, \gamma=0}^{\eta_{\alpha, \eta} \eta_{\beta}, \eta_{\gamma}} f^{\alpha \beta \gamma} \ell_{\alpha}(\xi) \ell_{\beta}(\eta) \ell_{\gamma}(\zeta),
\end{gathered}
$$

where $f^{\alpha \beta \gamma}=f\left(\mathbf{x}\left(\xi_{\alpha}, \eta_{\beta}, \zeta_{\gamma}\right)\right)$ denotes the value of the function $f$ at the GLL point $\mathbf{x}\left(\xi_{\alpha}, \eta_{\beta}, \zeta_{\gamma}\right)$. To simplify the notation, we omit the degree $n$ as a superscript on the Lagrange polynomials. Using the polynomial representation (50), the gradient of a function, $\nabla f=\sum_{i=1}^{3} \hat{\mathbf{x}}_{i} \partial_{i} f$, evaluated at the GLL point $\mathbf{x}\left(\xi_{\alpha}, \eta_{\beta}, \zeta_{\gamma^{\prime}}\right)$, can be expressed as

$$
\begin{aligned}
\nabla f\left(\mathbf{x}\left(\xi_{\alpha^{\prime}}, \eta_{\beta^{\prime}}, \zeta_{\gamma^{\prime}}\right)\right) \\
\approx \sum_{i=1}^{3} \hat{\mathbf{x}}_{i}\left[\left(\partial_{i} \xi\right)^{\alpha^{\prime} \beta^{\prime} \gamma^{\prime}} \sum_{\alpha=0}^{\eta_{\alpha}} f^{\alpha \beta^{\prime} \gamma^{\prime}} \ell_{\alpha}^{\prime}\left(\xi_{\alpha^{\prime}}\right)\right. \\
+\left(\partial_{i} \eta\right)^{\alpha^{\prime} \beta^{\prime} \gamma^{\prime}} \sum_{\beta=0}^{\eta_{\beta}} f^{\alpha^{\prime} \beta \gamma^{\prime} \ell_{\beta}^{\prime}\left(\eta_{\beta^{\prime}}\right)} \\
\left.+\left(\partial_{i} \zeta\right)^{\alpha^{\prime} \beta^{\prime} \gamma^{\prime}} \sum_{\gamma=0}^{\eta_{\gamma}} f^{\alpha^{\prime} \beta^{\prime} \gamma} \ell_{\gamma}^{\prime}\left(\zeta_{\gamma^{\prime}}\right)\right]
\end{aligned}
$$

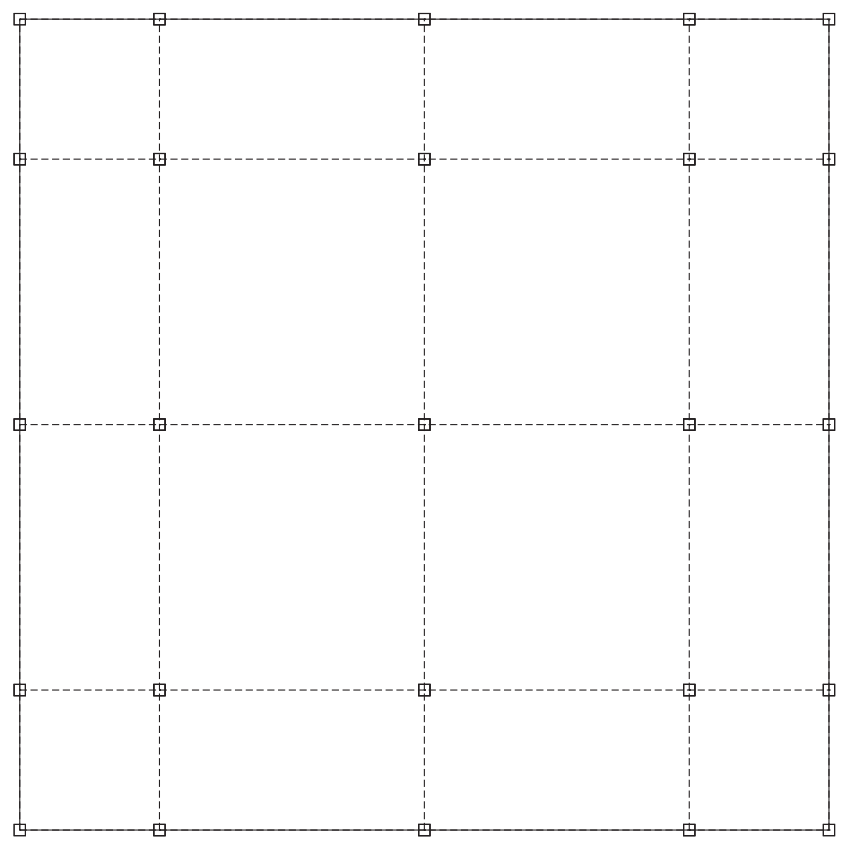

Figure 6. When a polynomial degree $n$ is used to discretize the wave field, each 3D spectral element contains a grid of $(n+1)^{3}$ Gauss-Lobatto-Legendre points, and each 2D face of an element contains a grid of $(n+1)^{2}$ Gauss-Lobatto-Legendre points, as illustrated here in the case of $n=4$. These points are non-evenly spaced. Low-order finite-element methods usually use $n=1$ or $n=2$, while in the spectral-element method for seismic wave propagation $n$ is usually chosen between 4 and 10 . 
Here $\hat{\mathbf{x}}_{i}, i=1,2,3$, denote unit vectors in the direction of increasing $x, y$, and $z$, respectively, and $\partial_{i}, i=1,2,3$, denote partial derivatives in those directions. We use a prime to denote derivatives of the Lagrange polynomials, as in $\ell_{\alpha}^{\prime}$. The matrix $\partial \xi / \partial \mathbf{x}$ is obtained by inverting the matrix $\partial \mathbf{x} /$ $\partial \xi$. We know that this inverse exists, because this is a requirement that we imposed during mesh design (the Jacobian never vanishes).

\subsection{Numerical Integration}

To solve the weak form of the equation of motion (19), integrals need to be evaluated numerically over each element. In the context of classical FEMs, one frequently uses Gauss quadrature for this purpose. In a SEM, a GaussLobatto-Legendre (GLL) integration rule is used instead, because it leads to a diagonal mass matrix when used in conjunction with GLL interpolation points. Integrations over elements with volume $\Omega_{e}$ hence give

$$
\begin{array}{rl}
\int_{\Omega_{e}} & f(\mathbf{x}) \mathrm{d}^{3} \mathbf{x} \\
& =\int_{-1}^{1} \int_{-1}^{1} \int_{-1}^{1} f(\mathbf{x}(\xi, \eta, \zeta)) J(\xi, \eta, \zeta) \mathrm{d} \xi \mathrm{d} \eta \mathrm{d} \zeta \\
& \approx \sum_{\alpha, \beta, \gamma=0}^{\eta_{\alpha}, \eta_{\beta}, \eta \gamma} \omega_{\alpha} \omega_{\beta} \omega_{\gamma} f^{\alpha \beta \gamma} J^{\alpha \beta \gamma},
\end{array}
$$

where $J^{\alpha \beta \gamma}=J\left(\xi_{\alpha}, \eta_{\beta}, \zeta_{\gamma}\right)$, and $\omega_{\alpha}>0$, for $\alpha=0, \ldots, n$, denote the weights of the GLL quadrature [Canuto et al., 1988].

On the fluid-solid boundaries in the model we need to evaluate surface integrals in order to implement the coupling based upon domain decomposition. At the elemental level, using GLL quadrature, these surface integrations may be written in the form

$$
\begin{aligned}
\int_{\Gamma_{b}} f(\mathbf{x}) \mathrm{d}^{2} \mathbf{x} & =\int_{-1}^{1} \int_{-1}^{1} f(\mathbf{x}(\xi, \eta)) J_{b}(\xi, \eta) \mathrm{d} \xi \mathrm{d} \eta \\
& \approx \sum_{\alpha, \beta=0}^{\eta_{\alpha}, \eta_{\beta}} \omega_{\alpha} \omega_{\beta} f^{\alpha \beta} J_{b}^{\alpha \beta},
\end{aligned}
$$

where $\Gamma_{b}$ denotes a surface element located on the fluidsolid interface, and $J_{b}^{\alpha \beta}=J_{b}\left(\xi_{\alpha}, \eta_{\beta}\right)$ is the surface Jacobian (5) evaluated at the GLL points. Note that in local and regional simulations the implementation of the absorbing boundary conditions represented by the last term in (19) also involves numerical integrations of the form (53) along the edges of the model.
Other authors [e.g., Priolo et al., 1994; Seriani, 1998] use a different implementation of the SEM based on Chebyshev polynomials. The main advantages are that the Gauss-Lobatto-Chebyshev integration rule is exact for the chosen polynomial basis, while it is only approximate in the case of GLL (see Section 3.11), and that the Gauss-LobattoChebyshev points and weights are known analytically (in the GLL version they are computed numerically). The main disadvantage of the approach is that it does not lead to a diagonal mass matrix, and that therefore iterative solvers for large matrix systems have to be implemented and an implicit time-marching scheme is often used. This is technically difficult, but has been used successfully even in 3-D [Seriani, 1997, 1998]. Because an unconditionally stable implicit time scheme is used, this method is not sensitive to very high Pwave speeds in small grid cells that can drastically reduce the time step and therefore increase the cost of the explicit time scheme used in the Legendre approach.

\subsection{Discrete Form of the Weak Equation in the Solid Regions}

In the weak form of the solid wave equations (19), (22), (43), and (47), we first need to expand the displacement field $\mathbf{s}$ in terms of Lagrange polynomials:

$$
\mathbf{s}(\mathbf{x}(\xi, \eta, \zeta), t) \approx \sum_{i=1}^{3} \hat{\mathbf{x}}_{i} \sum_{\sigma, \tau, v=0}^{\eta_{\sigma,}, \eta_{\tau}, \eta_{v}} s_{i}^{\sigma \tau v}(t) \ell_{\sigma}(\xi) \ell_{\tau}(\eta) \ell_{v}(\zeta)
$$

and the test vector $\mathbf{w}$ :

$\mathbf{w}(\mathbf{x}(\xi, \eta, \zeta))=\sum_{i=1}^{3} \hat{\mathbf{x}}_{i} \sum_{\sigma, \beta, \gamma=0}^{\eta_{\sigma,} \eta_{\tau}, \eta_{v}} w^{\alpha \beta \gamma} \ell_{\alpha}(\xi) \ell_{\beta}(\eta) \ell_{\gamma}(\zeta)$

Because we use the same basis functions to express the displacement and test vectors, the SEM is a so-called Galerkin method. The next step is to evaluate the integrals at the elemental level based upon GLL quadrature. At the elemental level, for the first term on the left-hand side of the equations of motion (19), (22), (43), and (47), known as the mass matrix, this integration gives

$$
\begin{aligned}
\int_{\Omega_{e}} \rho \mathbf{w} \cdot \partial_{t}^{2} \mathbf{s} \mathrm{d}^{3} \mathrm{x}= & \int_{-1}^{1} \int_{-1}^{1} \int_{-1}^{1} \rho(\mathbf{x}(\boldsymbol{\xi})) \\
& \mathbf{w}(\mathbf{x}(\boldsymbol{\xi})) \cdot \partial_{t}^{2} \mathbf{s}(\mathbf{x}(\boldsymbol{\xi}), t) J(\boldsymbol{\xi}) \mathrm{d}^{3} \boldsymbol{\xi} .
\end{aligned}
$$

Upon substituting the interpolations (54) and (55) in (56), using the quadrature (52), we obtain 


$$
\begin{aligned}
\int_{\Omega_{e}} \rho \mathbf{w} \cdot \partial_{t}^{2} \mathbf{s} \mathrm{d}^{3} \mathbf{x} \approx & \sum_{\alpha, \beta, \gamma=0}^{\eta_{\alpha}, \eta_{\beta}, \eta_{\tau}} \omega_{\alpha} \omega_{\beta} \omega_{\gamma} J^{\alpha \beta \gamma} \rho^{\alpha \beta \gamma} \\
& \times \sum_{i=1}^{3} w_{i}^{\alpha \beta \rho} \ddot{s}_{i}^{\alpha \beta \gamma}(t)
\end{aligned}
$$

where $\rho^{\alpha \beta \gamma}=\rho\left(\mathbf{x}\left(\xi_{\alpha}, \eta_{\beta}, \zeta_{\gamma}\right)\right)$, and where a dot denotes differentiation with respect to time. By independently setting factors of $w_{1}^{\alpha \beta \gamma}, w_{2}^{\alpha \beta \gamma}$, and $w_{3}^{\alpha \beta \gamma}$ equal to zero, since the weak formulation (19) must hold for any test vector $\mathbf{w}$, we obtain independent equations for each component of acceleration $\ddot{s}_{i}^{\alpha \beta \gamma}(t)$ at grid point $\left(\xi_{\alpha}, \eta_{\beta}, \zeta_{\gamma}\right)$. One can see that the value of acceleration at each point of a given element $\ddot{s}_{i}^{\alpha \beta \gamma}(t)$ is simply multiplied by the factor $\omega_{\alpha} \omega_{\beta} \omega_{\gamma} \rho^{\alpha \beta \gamma} J^{\alpha \beta \gamma}$, which means that the elemental mass matrix is exactly diagonal. This is one of the key ideas behind the SEM, and the main motivation behind the choice of Lagrange interpolation at the GLL points used in conjunction with GLL numerical integration.

The weak form of the Coriolis term in (22), (43), and (47) for an element $\Omega_{e}$ is

$$
\begin{aligned}
& \int_{\Omega_{e}} 2 \rho \mathbf{w} \cdot\left(\boldsymbol{\Omega} \times \partial_{t} \mathbf{s}\right) \mathrm{d}^{3} \mathbf{r} \approx \\
& 2 \Omega \sum_{\alpha, \beta, \gamma=0}^{n} \omega_{\alpha} \omega_{\beta} \omega_{\gamma} J^{\alpha \beta \gamma} \rho^{\alpha \beta \gamma} \sum_{i, j=1}^{3} w_{i}^{\alpha \beta \gamma} \epsilon_{i 3 j} \dot{s}_{j}^{\alpha \beta \gamma} .
\end{aligned}
$$

Here $\epsilon_{i j k}$ denotes the alternating tensor. It is worth mentioning that the effect of rotation has a negligible impact on the numerical cost of realistic global Earth simulations, both in terms of memory requirements and CPU time, because, considering that rotation is a small effect, the Coriolis term (58) can be handled based on an explicit time scheme and therefore added to the right-hand side of the wave equation after discretization at the global level.

The first integral on the right-hand side of (19), (22), (43), and (47) that needs to be evaluated at the elemental level is the so-called stiffness matrix. We can write

$$
\begin{aligned}
\int_{\Omega_{e}} \nabla \mathbf{w}: \mathbf{T} \mathrm{d}^{3} \mathbf{x} \approx \sum_{\alpha, \beta, \gamma=0}^{\eta_{\alpha}, \eta_{\beta^{\prime}}, \eta \gamma} \sum_{i=1}^{3} w_{i}^{\alpha \beta \gamma} \\
\times\left[\omega_{\beta} \omega_{\gamma} \sum_{\alpha^{\prime}=0}^{\eta_{\alpha^{\prime}}} \omega_{\alpha^{\prime}} J_{e}^{\alpha^{\prime} \beta \gamma} F_{i 1}^{\alpha^{\prime} \beta \gamma} \ell_{\alpha}^{\prime}\left(\xi_{\alpha^{\prime}}\right)\right. \\
+\omega_{\alpha} \omega_{\gamma} \sum_{\beta=0}^{\eta_{\beta^{\prime}}} \omega_{\beta^{\prime}} J_{e}^{\alpha \beta^{\prime} \gamma} F_{i 2}^{\alpha \beta^{\prime} \gamma} \ell_{\beta}^{\prime}\left(\eta_{\beta^{\prime}}\right) \\
\left.+\omega_{\alpha} \omega_{\beta} \sum_{\gamma^{\prime}=0}^{\eta \gamma^{\prime}} \omega_{\gamma^{\prime}} J_{e}^{\alpha \beta \gamma^{\prime}} F_{i 3}^{\alpha \beta \gamma^{\prime}} \ell_{\gamma}^{\prime}\left(\zeta_{\gamma^{\prime}}\right)\right] .
\end{aligned}
$$

where

$$
F_{i k}=\sum_{j=1}^{3} T_{i j} \partial_{j} \xi_{k},
$$

and where $F_{i k}^{\sigma \tau v}=F_{i k}\left(\mathbf{x}\left(\xi_{\sigma}, \eta_{\tau}, \zeta_{v}\right)\right)$ denotes the value of $F_{i k}$ at the GLL point $\mathbf{x}\left(\xi_{\sigma}, \eta_{\tau}, \zeta_{v}\right)$. For brevity, we have introduced index notation $\xi_{i}, \mathrm{i}=1,2,3$, where $\xi_{1}=\xi, \xi_{2}=\eta$, and $\xi_{3}=\zeta$. In this notation, the elements of the mapping matrix $\partial \xi / \partial \mathbf{x}$ may be written as $\partial_{i} \xi_{j}$. In the case of selfgravitation, in (59) the matrix $\mathbf{T}$ needs to be replaced by $\mathbf{T}+\mathbf{G}$, as in (22), (43), and (47).

The value of the stress tensor $\mathbf{T}$ at the integration points is determined by

$$
\begin{aligned}
\mathbf{T}\left(\mathbf{x}\left(\xi_{\alpha}, \eta_{\beta}, \zeta_{\gamma}\right), t\right)= \\
\mathbf{c}\left(\mathbf{x}\left(\xi_{\alpha}, \eta_{\beta}, \zeta_{\gamma}\right)\right): \nabla \mathbf{s}\left(\mathbf{x}\left(\xi_{\alpha}, \eta_{\beta}, \zeta_{\gamma}\right), t\right)
\end{aligned}
$$

Note that, as mentioned in the discussion after (8), we have made no particular assumption on the structure of the stiffness tensor c, i.e., the SEM can handle a fully anisotropic tensor with 21 independent coefficients (see also Komatitsch et al.[2000b] and Komatitsch and Tromp [2002a]). In our SEM implementation, the ratio of CPU time requirements with full anisotropy with 21 coefficients compared to an isotropic simulation is approximately 1.15 . To perform the calculation (61), we first need to compute the gradient of displacement $\nabla \mathbf{s}$ at the GLL integration points. Upon differentiating (54) we get

$$
\begin{aligned}
\partial_{i} s_{j}\left(\mathbf{x}\left(\xi_{\alpha}, \eta_{\beta}, \zeta_{\gamma}\right), t\right)= \\
\left.+\sum_{\sigma=0}^{n_{\alpha}} s_{j}^{\alpha \beta \gamma}(t) \ell_{\sigma}^{\prime}\left(\xi_{\alpha}\right)\right] \partial_{i} \xi\left(\xi_{\alpha}, \eta_{\beta}, \zeta_{\gamma}\right) \\
+\left[\sum_{\sigma=0}^{n_{\alpha}} s^{\alpha \sigma \gamma}(t) \ell_{\sigma}^{\prime}\left(\eta_{\beta}\right)\right] \partial_{i} \eta\left(\xi_{\alpha}, \eta_{\beta}, \zeta_{\gamma}\right), j \\
+\left[\sum_{\sigma=0}^{n_{\sigma}} s_{j}^{\alpha \beta \sigma}(t) \ell_{\sigma}^{\prime}\left(\zeta_{\gamma}\right)\right] \partial_{i} \zeta\left(\xi_{\alpha}, \eta_{\beta}, \zeta_{\gamma}\right) .
\end{aligned}
$$

In an anelastic medium, the stiffness matrix is still defined by (59), except that the stress tensor (61) is

$$
\begin{aligned}
\mathbf{T}\left(\mathbf{x}\left(\xi_{\alpha}, \eta_{\beta}, \zeta_{\gamma}\right), t\right) & =\mathbf{c}\left(\mathbf{x}\left(\xi_{\alpha}, \eta_{\beta}, \zeta_{\gamma}\right)\right): \nabla \mathbf{s}\left(\mathbf{x}\left(\xi_{\alpha}, \eta_{\beta}, \zeta_{\gamma}\right), t\right) \\
& -\sum_{\ell=1}^{L} \mathbf{R}_{\ell}\left(\mathbf{x}\left(\xi_{\alpha}, \eta_{\beta}, \zeta_{\gamma}\right), t\right)
\end{aligned}
$$

in accordance with (11). This implies that the five independent components of the symmetric, zero-trace memory tensor $\mathbf{R}_{\ell}$ need to be stored on the grid for each standard linear 
solid. Because of this requirement, anelastic simulations require significantly more memory than purely elastic calculations. In our SEM implementation based on three linear solids in parallel, the ratio of memory requirements and of CPU time requirements with and without anelasticity is approximately 1.5 . To reduce these additional requirements, one can spread the memory variables across adjacent grid points to mimic the expected anelastic behavior in average [Zeng, 1996; Day, 1998].

The second term on the right-hand side of the weak form of the equation of motion (19), (22), and (47) is the source term, which may be expressed as

$$
\begin{aligned}
& \int_{\Sigma_{s}} \mathbf{m}\left(\mathbf{x}_{\mathrm{s}}, t\right): \nabla \mathbf{w}\left(\mathbf{x}_{\mathrm{s}}\right) \mathrm{d}^{2} \mathbf{x}_{\mathrm{s}} \approx \sum_{i=1}^{3} w_{i}^{\alpha \beta \gamma} \\
& \times\left[\omega_{\beta} \sum_{\alpha_{s}=0}^{\eta_{\alpha}} \omega_{\alpha_{s}} J_{b}^{\alpha_{s} \beta} g_{i 1}^{\alpha_{s} \beta \gamma} \ell_{\alpha}^{\prime}\left(\xi_{\alpha_{s}}\right)\right. \\
& +\omega_{\alpha} \sum_{\beta_{s}=0}^{\eta_{\beta}} \omega_{\beta_{s}} J_{b}^{\alpha \beta_{s}} g_{i 2}^{\alpha \beta_{s} \gamma} \ell_{\beta}^{\prime}\left(\eta_{\beta_{s}}\right)
\end{aligned}
$$

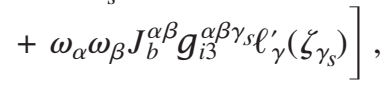

where

$$
g_{i k}=\sum_{j=1}^{3} m_{i j} \partial_{j} \xi_{k}
$$

For the point source (16) this reduces to

$$
\begin{aligned}
\mathbf{M}: & \nabla \mathbf{w}\left(\mathbf{x}_{\mathrm{s}}\right) \approx \sum_{\alpha, \beta, \gamma=0}^{\eta_{\alpha, \eta_{\beta}, \eta} \eta_{i=1}^{3}} w_{i}^{\alpha \beta \gamma} \\
& \left\{\sum_{\sigma, \tau, \nu=0}^{\eta_{\sigma,} \eta_{\tau}, \eta_{\nu}} \ell_{\sigma}\left(\xi_{\alpha_{s}}\right) \ell_{\tau}\left(\eta \beta_{\mathrm{s}}\right) \ell_{\nu}\left(\zeta_{\gamma_{s}}\right)\right. \\
& \times\left[G_{i 1}^{\sigma \tau \nu} \ell_{\alpha}^{\prime}\left(\xi_{\alpha_{s}}\right) \ell_{\beta}\left(\eta \beta_{s}\right) \ell_{\gamma}\left(\zeta_{\gamma_{s}}\right)\right. \\
& +G_{i 2}^{\sigma \tau \nu} \ell_{\alpha}\left(\xi_{\alpha_{s}}\right) \ell_{\beta}^{\prime}\left(\eta \beta_{s}\right) \ell_{\gamma}\left(\zeta_{\beta_{s}}\right) \\
& \left.\left.+G_{i 3}^{\sigma \tau \nu} \ell_{\alpha}\left(\xi_{\alpha_{s}}\right) \ell_{\beta}\left(\eta \beta_{s}\right) \ell_{\gamma}^{\prime}\left(\zeta_{\gamma_{s}}\right)\right]\right\},
\end{aligned}
$$

where we have defined $G_{i k}=\sum_{j=1}^{3} M_{i j} \partial_{j} \xi_{k}$, and where $G_{i k}^{\sigma \tau \nu}=G_{i k}\left(\mathbf{x}\left(\xi_{\sigma}, \eta_{\tau}, \zeta_{v}\right)\right)$ and $\mathbf{x}\left(\xi_{\alpha_{s}}, \eta_{\beta s}, \zeta_{\gamma_{s}}\right)=\mathbf{x}_{\mathrm{s}}$.

Note that unlike many grid methods, in the context of the SEM the source can be located anywhere in the model, and does not need to correspond with a GLL grid point. Because of the polynomial basis used in each element, a point moment-tensor source gets spread over the entire element that contains the point source after discretization. This is consistent with the use of a polynomial basis and is not a problem, except perhaps when receivers are placed in the same spectral element as the source [Faccioli et al., 1997], a situation almost never encountered in practice.

Self-gravitation in the context of the Cowling approximation contributes two terms to (22), (43), and (47). As mentioned earlier, the first contribution can be incorporated in the calculation of the stiffness matrix by making the substitution $\mathbf{T} \rightarrow \mathbf{T}+\mathbf{G}$ in (61), remembering that $\mathbf{G}$ is non-symmetric. The second gravity contribution has the weak form

$$
\begin{aligned}
\int_{\Omega_{e}} \rho \mathbf{s} \cdot \mathbf{H} \cdot \mathbf{w} \mathrm{d}^{3} \mathbf{r} \approx & \sum_{\alpha, \beta, \gamma=0}^{n} \omega_{\alpha} \omega_{\beta} \omega_{\gamma} J^{\alpha \beta \gamma} \rho^{\alpha \beta \gamma} \\
& \sum_{i, j=1}^{3} w_{i}^{\alpha \beta \gamma} H_{i j}^{\alpha \beta \gamma} s_{j}^{\alpha \beta \gamma} .
\end{aligned}
$$

Note that this gravity term is diagonal. The self-gravitation terms require no significant memory storage and have a minor impact on CPU time.

\subsection{Discrete Form of the Weak Equation in the Fluid Regions}

To obtain explicit expressions for the weak formulation of the problem in the fluid regions, we first expand the potential $\chi$ in terms of Lagrange polynomials:

$$
\chi(\mathbf{x}(\xi, \eta, \zeta), t) \approx \sum_{\sigma, \tau, \nu=0}^{\eta_{\sigma,} \eta_{\tau,} \eta_{\nu}} \chi^{\sigma \tau \nu}(t) \ell_{\sigma}(\xi) \ell_{\tau}(\eta) \ell_{\nu}(\zeta)
$$

and the scalar test function $w$ :

$$
w(\mathbf{x}(\xi, \eta, \zeta))=\sum_{\alpha, \beta, \gamma=0}^{\eta_{\sigma}, \eta_{\tau}, \eta_{\nu}} w^{\alpha \beta \gamma} \ell_{\alpha}(\xi) \ell_{\beta}(\eta) \ell_{\gamma}(\zeta) .
$$

Using this representation, the first term on the left-hand side of the weak form of the outer-core wave equation (33) may be written at the elemental level in the form

$$
\begin{gathered}
\int_{\Omega_{e}} \kappa^{-1} \rho w \partial_{t}^{2} \chi \mathrm{d}^{3} \mathbf{r} \approx \sum_{\alpha, \beta, \gamma=0}^{\eta \alpha, \eta_{\beta}, \eta_{\gamma}} \omega_{\alpha} \omega_{\beta} \omega_{\gamma} J^{\alpha \beta \gamma} \\
\left(\kappa^{\alpha \beta \gamma}\right)^{-1} \rho^{\alpha \beta \gamma} w^{\alpha \beta \gamma} \ddot{\chi}^{\alpha \beta \gamma}(t) .
\end{gathered}
$$

As for the left-hand side of the weak form in the solid regions, this elemental 'mass' matrix is diagonal.

The first integral on the right-hand side of the fluid weak formulation (33) becomes at the elemental level 


$$
\begin{aligned}
\int_{\Omega_{e}} \nabla w \cdot \nabla \chi \mathrm{d}^{3} \mathbf{r} \approx \sum_{\alpha, \beta, \gamma=0}^{\eta_{\alpha}, \eta_{\beta}, \eta_{\gamma}} w^{\alpha \beta \gamma} \\
\quad\left[\omega_{\beta} \omega_{\gamma} \sum_{\alpha^{\prime}=0}^{\eta_{\alpha^{\prime}}} \omega_{\alpha^{\prime}} J_{e}^{\alpha^{\prime} \beta \gamma}\left(\partial_{1} \chi\right)^{\alpha^{\prime} \beta \gamma} \ell_{\alpha}^{\prime}\left(\xi_{\alpha^{\prime}}\right)\right. \\
+\omega_{\alpha} \omega_{\gamma} \sum_{\beta^{\prime}=0}^{\eta_{\beta^{\prime}}} \omega_{\beta^{\prime}} J_{e}^{\alpha \beta^{\prime} \gamma}\left(\partial_{2} \chi\right)^{\alpha \beta^{\prime} \gamma} \ell_{\beta}^{\prime}\left(\eta_{\beta^{\prime}}\right) \\
+\omega_{\alpha} \omega_{\beta} \sum_{\gamma^{\prime}=0}^{\eta_{\gamma^{\prime}}} \omega_{\gamma^{\prime}} J_{e}^{\alpha \beta \gamma^{\prime}}\left(\partial_{3} \chi\right)^{\left.\alpha \beta \gamma^{\prime} \ell^{\prime}\left(\zeta_{\gamma^{\prime}}\right)\right]}
\end{aligned}
$$

where

$$
\begin{aligned}
\left(\partial_{i} \chi\right)^{\alpha \beta \rho} & =\sum_{\alpha=0}^{\eta_{\alpha}} \chi^{\alpha \beta^{\prime} \gamma^{\prime}} \ell_{\alpha}^{\prime}\left(\xi_{\alpha^{\prime}}\right) \partial_{i} \xi \\
& +\sum_{\beta=0}^{\eta_{\beta}} \chi^{\alpha^{\prime} \beta \gamma^{\prime}} \ell_{\beta}^{\prime}\left(\eta_{\beta^{\prime}}\right) \partial_{i} \eta \\
& +\sum_{\gamma=0}^{\eta_{\gamma}} \chi^{\alpha^{\prime} \beta^{\prime} \gamma} \ell_{\gamma}^{\prime}\left(\zeta_{\gamma^{\prime}}\right) \partial_{i} \zeta .
\end{aligned}
$$

The second term on the right-hand side of the weak form of the wave equation in the outer core (33), which arises in the presence of self-gravitation and rotation, is

$$
\begin{gathered}
\int_{\Omega_{e}} \kappa^{-1} \rho w \mathbf{g} \cdot(\nabla \chi+\mathbf{u}) d^{3} \mathbf{r} \approx \\
\sum_{\alpha, \beta, \gamma=0}^{n} \omega_{\alpha} \omega_{\beta} \omega_{\gamma} J^{\alpha \beta \gamma} w^{\alpha \beta \gamma}\left(\kappa^{\alpha \beta \gamma}\right)^{-1} \rho^{\alpha \beta \gamma} \\
\sum_{i=1}^{3} g_{i}^{\alpha \beta \gamma}\left[\left(\partial_{i} \chi\right)^{\alpha \beta \gamma}+u_{i}^{\alpha \beta \gamma}\right]
\end{gathered}
$$

where $\left(\partial_{i} \chi\right)^{\alpha \beta \gamma}$, and where we have made the Cowling approximation.

The weak form of (34) is, in fact, entirely diagonal, which means that one needs to solve a second-order ordinary differential equation at each GLL point. Making the Cowling approximation and assuming that $N^{2}=0$ reduces the problem to solving the first-order equation (36), which may be accomplished based upon a Runge-Kutta scheme, as discussed in Komatitsch and Tromp [2002b].

\subsection{Coupling Between Fluid and Solid Regions}

The final term on the right-hand side of the weak forms of the equation of motion in the solid regions (22), (43), and (47) is the surface integral over the CMB or ICB that represents the interactions in traction and normal velocity between the solid mantle, the liquid core, and the solid inner core. A key ingredient of our domain decomposition technique is that, since we have a conforming mesh everywhere, i.e., the grid points on the $\mathrm{CMB}$ are common to the meshes in the mantle and in the outer core and the grid points on the ICB are common to the meshes in the outer and inner core, we can take the value of pressure at a given grid point from the fluid side and use it directly in the surface integral on the solid side. Therefore, no interpolation is needed at a fluidsolid interface. This type of matching is referred to as pointwise matching in the finite-element literature. At the elemental level on a boundary, the surface integrals over the CMB and the ICB in the solid regions may therefore be expressed as

$$
\int p \hat{\mathbf{n}} \cdot \mathbf{w d}^{2} \mathbf{x} \approx \sum_{\alpha, \beta=0}^{\eta_{\alpha}, \eta_{\beta}} \omega_{\alpha} \omega_{\beta} J_{b}^{\alpha \beta} p^{\alpha \beta}(t) \sum_{i=1}^{3} \omega_{i}^{\alpha \beta} \hat{n}_{i}^{\alpha \beta},
$$

where $J_{b}$ is the surface Jacobian (5), $\hat{\mathbf{n}}$ is the normal (4), and where we calculate the pressure $p$ based upon (37), taking the values of the right-hand side terms from the fluid side.

Similarly, the two surface integrals over the CMB and the ICB in the weak form of the equation of motion in the outer core (33) may be expressed as

$$
\int w \hat{\mathbf{n}} \cdot \mathbf{s} \mathrm{d}^{2} \mathbf{x} \approx \sum_{\alpha, \beta=0}^{\eta_{\alpha}, \eta_{\beta}} \omega_{\alpha} \omega_{\beta} J_{b}^{\alpha \beta} w^{\alpha \beta} \sum_{i=1}^{3} s_{i}^{\alpha \beta} \hat{n}_{i}^{\alpha \beta}
$$

\subsection{Accuracy of the Method}

In a standard FEM, low-degree polynomials (usually of degree 1 or 2) are used to discretize functions, and therefore the accuracy of the method can mainly be adjusted based upon the typical size of an element in the mesh, $\Delta h$, i.e., based upon mesh density. This means that in a traditional FEM, mesh design is the main parameter that controls accuracy. In a SEM, however, high-degree Lagrange interpolants are used to express functions. Therefore, the polynomial degree used to represent functions on an element, $n$, is an additional parameter that can be used to adjust the accuracy of the method.

Even on a unit cube with homogeneous material properties, the GLL numerical integration rule is exact only for polynomials of degree $2 n-1$. Any integration on the reference element involving the product of two polynomials of degree $n$-the displacement and the test function- is never exact, even in this simplest case. For deformed elements there are additional errors related to curvature [Maday and Rønquist, 1990]; the same is true for elements with heteroge- 
neous material properties. Thus a diagonal mass matrix is obtained in the Legendre SEM by purposely selecting a numerical integration rule that is not exact (but of course still very accurate) for the polynomial basis chosen (a process known as sub-integration in the finite-element literature). In this respect, the SEM is related to FEMs in which mass lumping is used to avoid the costly resolution of the nondiagonal system resulting from the use of a Gauss quadrature rule [e.g., Cohen et al., 1993]. As mentioned above, a different choice is made in the Chebyshev SEM used by some authors [e.g., Priolo et al., 1994; Seriani, 1998], in which an integration rule that is exact for the polynomial basis chosen is used, with the consequence that the exactly diagonal mass matrix is lost.

One of the shortcomings of the SEM for elastic media is that to our knowledge no theoretical analysis of its accuracy is available in the literature. For other classical numerical techniques such an analysis is usually performed in the spectral domain for a regular grid in a homogeneous medium. In the case of the SEM doing so turns out to be challenging because the polynomial degree used to discretize the wave field is high and also because the GLL numerical integration points are non-evenly spaced, which makes it technically difficult to use a standard Fourier analysis to perform the accuracy study, even in the case of a regular mesh of spectral elements in a homogeneous medium. In addition, one of the main ideas behind the use of the SEM is to take advantage of its geometrical flexibility and to therefore use highlydistorted non-regular meshes. On such meshes, performing an accuracy analysis is even more difficult because there are additional error terms associated with the distortion of the mesh elements, as in any finite element or spectral method [Maday and Rønquist, 1990]. To our knowledge, only Tordjman [1995] and Cohen and Fauqueux [2000] have attempted such a theoretical accuracy study for the SEM in the acoustic case, and Seriani and Priolo [1994] have addressed the issue based on a numerical study, also in the acoustic case.

Therefore, by trial and error, heuristic rules of thumb have emerged in order to determine how to select the polynomial degree to use in practice for an elastic SEM with a nonregular deformed mesh and a heterogeneous medium. Basically, we have used the main conclusions of Seriani and Priolo [1994] in the acoustic case and checked numerically by performing numerous benchmarks and comparisons to analytical solutions for simple cases that these conclusions extend reasonably well to the elastic case. Based on these numerical experiments, we can say that using polynomial degrees lower than typically 4 leads to similar inaccuracies as with standard FEMs [Marfurt, 1984], i.e., a large amount of numerical dispersion, which means that with such low degrees the advantages of using a SEM are lost. In contrast, if the polynomial degree is very large, e.g., greater than 10 , the SEM is spatially very accurate, but the computational requirements become prohibitive because of the size of the calculations related to matrix multiplications involving the full stiffness matrix, a process with a cost of $O\left(n^{4}\right)$ in 3-D, i.e., the numerical cost of the technique becomes prohibitive. Another problem in the case of a high degree is that the nonevenly spaced GLL numerical integration points become clustered toward the edges of each spectral element (the spacing between the first two GLL points varies approximately as $O\left(n^{-2}\right)$ ), and as a result of the small distance between these first two points, very small time steps have to be used to keep the explicit time-marching scheme stable (see Section 4), which drastically increases the cost of the Legendre SEM. Therefore, the rule of thumb is that for most wave propagation applications, polynomial degrees between approximately 4 and 10 should be used in practice.

In our case, we always use a polynomial degree $n=4$. In order to obtain accurate results, we use another heuristic rule of thumb that says that for this polynomial degree the average grid spacing $\Delta h$ should be chosen such that the average number of points per minimum wavelength $\lambda_{\min }$ in an element, $(n+1) \lambda_{\min } / \Delta h$, be roughly equal to 5 . It would be of interest to study this more precisely in the 3-D elastic case based on numerical experiments with different polynomial degrees and different values of the grid spacing, and to see how this accuracy analysis would compare to similar studies for more classical numerical techniques such as the finite-difference method. Such a comparative study should include both body and surface waves, the former being more difficult to model accurately than the latter, in particular for methods that are not based on a variational formulation of the wave equation. Schubert [2003] has started to study this problem in the 1-D case.

\section{TIME INTEGRATION OF THE GLOBAL SYSTEM}

In each individual spectral element, functions are sampled at the GLL points of integration. As can be seen in Figure 5, these points include -1 and 1, i.e., each element has grid points located exactly on its edges, and therefore shares these points (on its faces, edges, or corners) with neighboring elements in the spectral-element mesh. Therefore, as in a classical FEM, we need to distinguish the local mesh of grid points inside each element from the global mesh of points in the entire structure, which contains many points that are shared amongst several elements. In addition, the number of elements that share a given point (the so-called valence of a point) can vary and take any value in the mesh (in other words, the mesh can be non-structured), unlike in a regular mesh of cubes, in which the valence of a shared 
point is always 2 inside a face, 4 on an edge, and 8 at a corner.

Therefore, the first required step is to uniquely number the global points in order to define a mapping between the local mesh and the global mesh. This can be accomplished using efficient finite-element global numbering libraries that either take advantage of the known and fixed topology of the mesh (valence and list of neighbors) to uniquely assign a global point number to each local point inside a given spectral element, or perform a triple sorting algorithm on each coordinate of the points (sorting by increasing $x$, then by increasing $y$ for the same $x$, then by increasing $z$ for the same $x$ and $y$ ), again to detect common points and uniquely assign a global point number to each local point. Once this mapping has been defined, the internal forces computed separately on each element need to be summed at common grid points (in the finite-element literature this step is called 'assembling the system'). On a parallel computer, this part is the only step in the SEM that involves communications between adjacent mesh slices, as we will see in Section 5, because different slices located on different processors can share common points on their respective edges.

Let $U$ denote the global displacement vector in the solid regions of the model, i.e., $U$ contains the displacement vector at all the grid points in the global mesh, classically referred to as the global degrees of freedom of the system. The time evolution of the global system is governed by an ordinary differential equation of the general form

$$
M_{s} \ddot{U}+K_{s} U+B_{s} p=F_{s},
$$

where $M_{s}$ denotes the global diagonal mass matrix, $K_{s}$ the global stiffness matrix, $B_{s} p$ the fluid-solid coupling term involving the pressure $p$ that represents the boundary interactions at the CMB or ICB, and $F_{s}$ the earthquake source term. As mentioned above, one can take advantage of the fact that the global mass matrix is diagonal by using a fully explicit second-order finite-difference scheme to march this secondorder ordinary differential equation, moving the stiffness term to the right-hand side. The memory-variable equation (13) is solved for $\mathbf{R}_{\ell}$ using a modified second-order RungeKutta scheme in time, since such schemes are known to be efficient for this problem [Carcione, 1994]. We do not spread the memory variables across the grid.

In the fluid regions of the model (the outer core in the case of the global Earth), we similarly get an ordinary differential equation of the general form:

$$
M_{f} \ddot{X}+K_{f} X+B_{f} U=0,
$$

where $X$ is the global potential, $M_{f}$ denotes the global diagonal generalized mass matrix, $K_{f}$ the global generalized stiff- ness matrix, and $B_{f} U$ the global fluid-solid coupling term involving the solid displacements $U$ that represents the boundary interactions at the CMB or ICB (note that there is no source term in the fluid because the earthquake source is always located in the solid).

Compared to Komatitsch and Tromp [2002a, b], the improved formulation in the fluid region developed in Chaljub and Valette [2004] (see also Section 3.3 above) leads to a time-marching scheme in which there is no need to iterate on the fluid-solid coupling condition, i.e., following Chaljub and Valette [2004] we simply first solve (77) in the fluid and then (76) in the solid. The fluid-solid coupling term is evaluated based on the new values on the fluid side computed by solving (77).

The explicit time schemes introduced above are conditionally stable, i.e., for a given mesh and a given model there exists an upper limit on the time step above which calculations are unstable. One can define the Courant stability number of the explicit time integration schemes $C=\Delta t(v / \Delta h)_{\max }$, where $\Delta t$ is the time step chosen and $(v / \Delta h)_{\max }$ denotes the maximum ratio of $\mathrm{P}$-wave speed and grid spacing. The Courant stability condition [Courant et al., 1928] then says that the Courant number should not be chosen higher than an upper limit:

$$
C \leq C_{\max }
$$

that determines how large the time step can be while maintaining a stable simulation. Unfortunately, for the SEM to our knowledge there is no published theoretical analysis of how to determine the maximum Courant number $C_{\max }$. The heuristic rule of thumb that we use in practice is that for regular meshes $C_{\max } \simeq 0.5$, while for very irregular meshes with distorted elements and/or very heterogeneous media $C_{\max }$ reduces to approximately 0.3 to 0.4 . As for the issue of accuracy in Section 3.11, performing such a theoretical analysis for the SEM in the elastic case would be very difficult, even for a regular mesh in a homogeneous medium, because of the high polynomial degrees used, and because of the fact that the GLL numerical integration points are non-evenly spaced.

\section{IMPLEMENTATION ON PARALLEL COMPUTERS}

The mesh designed in Section 2 is too large to fit in memory on a single computer. Modern parallel computers such as clusters or grids of computers have a distributed memory architecture. The standard approach for programming parallel machines with distributed memory in a portable way is to use a message-passing methodology, usually based upon 
a library called MPI [e.g., Gropp et al., 1994], an acronym for 'Message Passing Interface'.

Because we use an explicit time-marching scheme (see Section 4), our SEM algorithm mostly consists in small local matrix-vector products in each spectral element, which implies that the processors spend most of their time doing actual calculations, and only a small amount of time in the communication step. Hence, the SEM algorithm is not very sensitive to the speed of the network connecting the different processors. It can therefore run on high-latency networks such as clusters of PC computers, sometimes referred to as 'Beowulf' machines, or on grids of computers.

In order to run an SEM algorithm on such parallel machines, we need to split the mesh into as many slices as the number of processors we use on the machine. Plate 1 shows how the mesh of Figure 3 for the global Earth is split into slices in the case of calculations distributed over 1944 processors ( 6 chunks of $18 \times 18$ slices each), as will be used in Section 6. Calculations can be performed locally by each processor on the spectral elements that constitute the mesh slice it carries, and one communication phase is then required at each time step of our time-marching algorithm in order to sum the internal forces computed at the common faces, edges, and corners shared by mesh slices carried by different processors. Therefore, MPI communication tables that orchestrate the (constant) sequence of messages that needs to be exchanged amongst the slices at each time step need to be created once and for all when the mesh is built.

\section{NUMERICAL RESULTS}

In previous work, synthetic seismograms for 3-D Earth models calculated based on the SEM have been successfully compared to broadband data, both for sedimentary basins [Komatitsch et al., 2004; Liu et al., 2004] and at a global scale [Komatitsch and Tromp, 2002b; Chaljub et al., 2003; Komatitsch et al., 2003; Tsuboi et al., 2003; Capdeville et al., 2003]. Here, as an application of the SEM to a largescale 3-D problem, we combine all the complications of a fully 3-D Earth model. The simulations include anisotropy (the reference 1-D PREM upper mantle model is anisotropic, see Komatitsch and Tromp [2002a] for details), attenuation, self-gravitation, the oceans, rotation, ellipticity, topography and bathymetry, a 3-D mantle model and a 3-D model of crustal wave speeds, as explained in Section 2 (see also Komatitsch and Tromp [2002b] for more details on these models).

The SEM calculations are performed on the Earth Simulator at the Japan Agency for Marine Earth Science and Technology (JAMSTEC). This computer has 640 8-processor compute nodes, for a total of 5120 processors. Each node has 16 gigabytes of shared memory, for a total of 10 terabytes of memory. The peak performance per node is 64 gigaflops (i.e., 64 billions of floating-point operations per second) and the total peak performance is 40 teraflops. On 48 nodes of the Earth Simulator we can model periods of $9 \mathrm{~s}$ and a typical simulation lasts 10 hours, 243 Earth Simulator nodes enable us to reach periods of $5 \mathrm{~s}$, and on 507 nodes this is further reduced to a shortest period of $3.5 \mathrm{~s}$ [Komatitsch et al., 2003; Tsuboi et al., 2003]. To put these numbers in perspective, typical normal-mode summation codes that calculate semi-analytical synthetic seismograms for 1-D Earth models are accurate down to $6 \mathrm{~s}$. In other words, the Earth Simulator allows us to simulate global seismic wave propagation in fully 3-D Earth models at periods shorter than current seismological practice for 1-D spherically symmetric models.

We model the September 2, 1997, $210 \mathrm{~km}$ deep Colombia earthquake, which had a magnitude of $M_{w}=6.7$. We use the mesh in Figures 3, 4 and Plate 1 and a polynomial degree $n=4$, which gives a grid composed of 82 million spectral elements and a total of 14.5 billion grid points. At the surface of the model the size of the spectral elements is $1.04^{\circ}$ in the two horizontal directions (i.e., the average spacing between adjacent grid points is $0.026^{\circ}$, or equivalently $2.9 \mathrm{~km}$ ). The time step is $\Delta t=72 \mathrm{~ms}$, and we propagate the signal for 3600 s. Receivers from the global network of seismic stations record the three components of displacement. Figure 7 shows the results of a simulation on the Earth Simulator accurate down to $5 \mathrm{~s}$. The source is the CMT solution taken from the Harvard catalog. The vertical component data are lined up on the Rayleigh wave. Note the remarkable fit both at short and long periods. In Figure 8 we present the same results centered on the P-wave arrival. Note the distinct $\mathrm{pP}$ and $\mathrm{sP}$ arrivals, and also note that the $\mathrm{sP}$ arrivals are consistently late in the SEM synthetic seismograms, which implies that the shear wave speed model we use is too slow in the mantle wedge above the subducting plate.

In the case of large earthquakes, the finite size of the earthquake source must be taken into account, and an equivalent CMT cannot be used. Plate 2 shows a snapshot of such a finite-fault simulation for the November 3, 2002, Denali fault, Alaska, earthquake, which had a magnitude of $M_{w}=$ 7.9 and ruptured several fault segments over a total distance of $220 \mathrm{~km}$ [Eberhart-Phillips et al., 2003]. The finite source model, which is represented by 475 point double couple solutions, was determined by Ji et al. [2004]. Because the earthquake rupture propagates in a southeasterly direction along the Denali fault, the waves that propagate along the West coast of the United States have large amplitudes. This directivity effect due to the finite size of the earthquake fault is captured well by the SEM simulations. We show full 

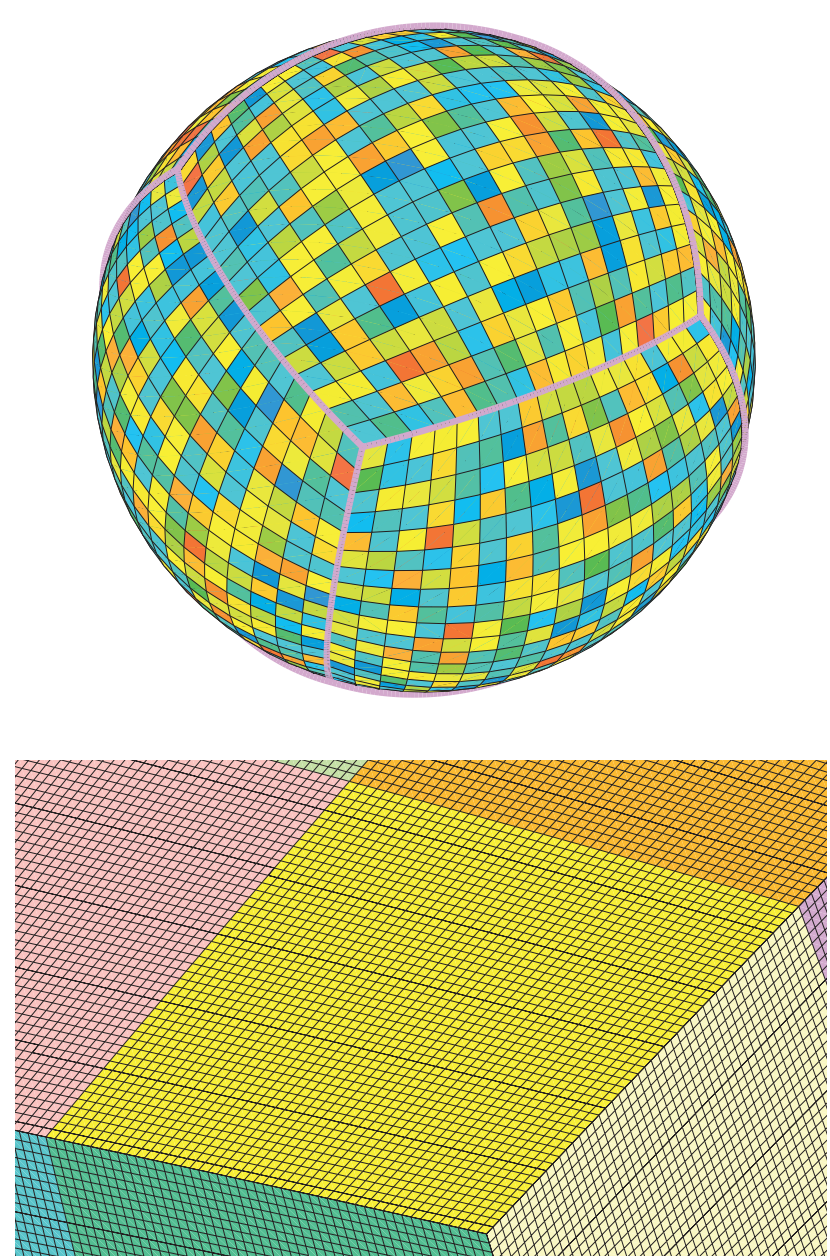

Plate 1. The spectral-element method uses a mesh of hexahedral finite elements on which the wave field is interpolated by highdegree Lagrange polynomials on Gauss-Lobatto-Legendre integration points. In order to perform the calculations on a parallel computer with distributed memory, the mesh in Figures 3 and 4 is split into slices based upon a regular domain-decomposition topology. Each slice is handled by a separate processor. Adjacent slices located on different processors exchange information about common faces and edges based upon a message-passing methodology. The top figure shows a global view of the mesh at the surface, illustrating that each of the six sides of the so-called 'cubed sphere' mesh is divided into $18 \times 18$ slices, shown here with different colors, for a total of 1944 slices. The bottom figure shows a closeup of the mesh of $48 \times 48$ spectral elements at the surface of each slice. Within each surface spectral element we use $5 \times 5=25$ GaussLobatto-Legendre grid points, which translates into an average grid spacing of $2.9 \mathrm{~km}$ (i.e., $0.026^{\circ}$ ) on the entire Earth surface.

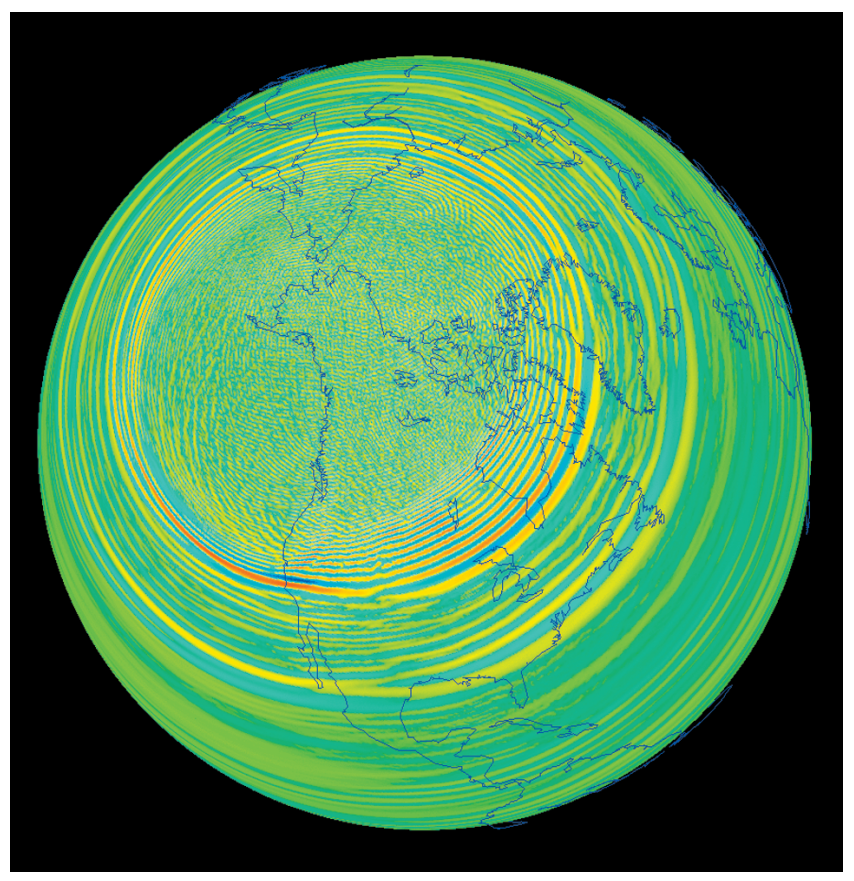

Plate 2. Snapshot of the propagation of seismic waves in the Earth generated by the November 3, 2002 Denali fault, Alaska, earthquake. Note the large amplification of the waves along the western coast of the United States. 


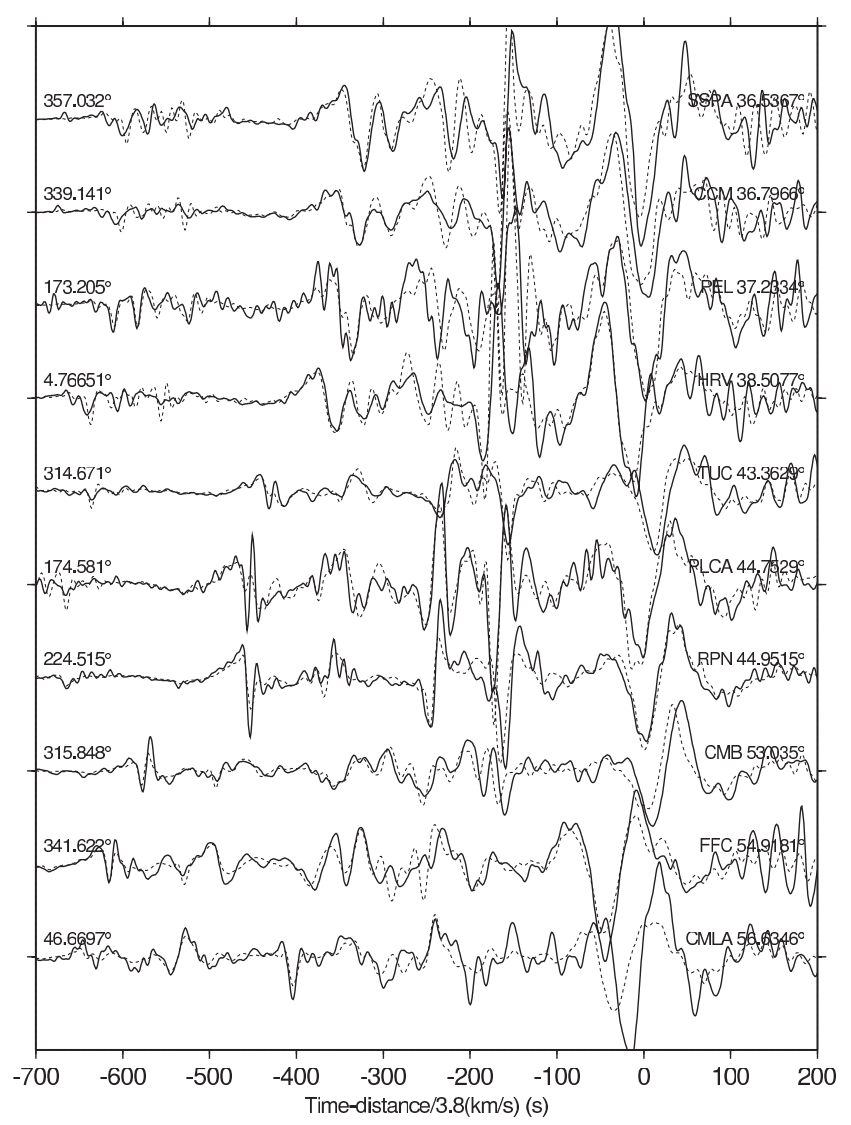

Figure 7. Comparison of vertical component data (solid line) and spectral-element synthetic seismograms (dashed line) for the September 2, 1997, $210 \mathrm{~km}$ deep Colombia earthquake. Both the synthetic seismograms and the data are lowpass-filtered at $5 \mathrm{~s}$. The source azimuth measured clockwise from due North is indicated on the left, and the station name and epicentral distance are on the right. Records are aligned on the Rayleigh wave.

waveform comparisons between data and synthetic seismograms in Figure 9. Note that the SEM synthetic seismograms capture the dispersion of the Rayleigh waves remarkably well.

\section{CONCLUSIONS}

We have presented a detailed description of the spectral element method for 3-D seismic wave propagation. The full complexity of Earth models, i.e., surface topography, attenuation, anisotropy and fluid-solid interfaces, and, in the case of global simulations, self-gravitation, rotation and the oceans, are taken into account. We have used an improved SEM formulation for the outer core in the presence of selfgravitation and rotation that has the important benefit that it does not require iterations on the fluid-solid coupling

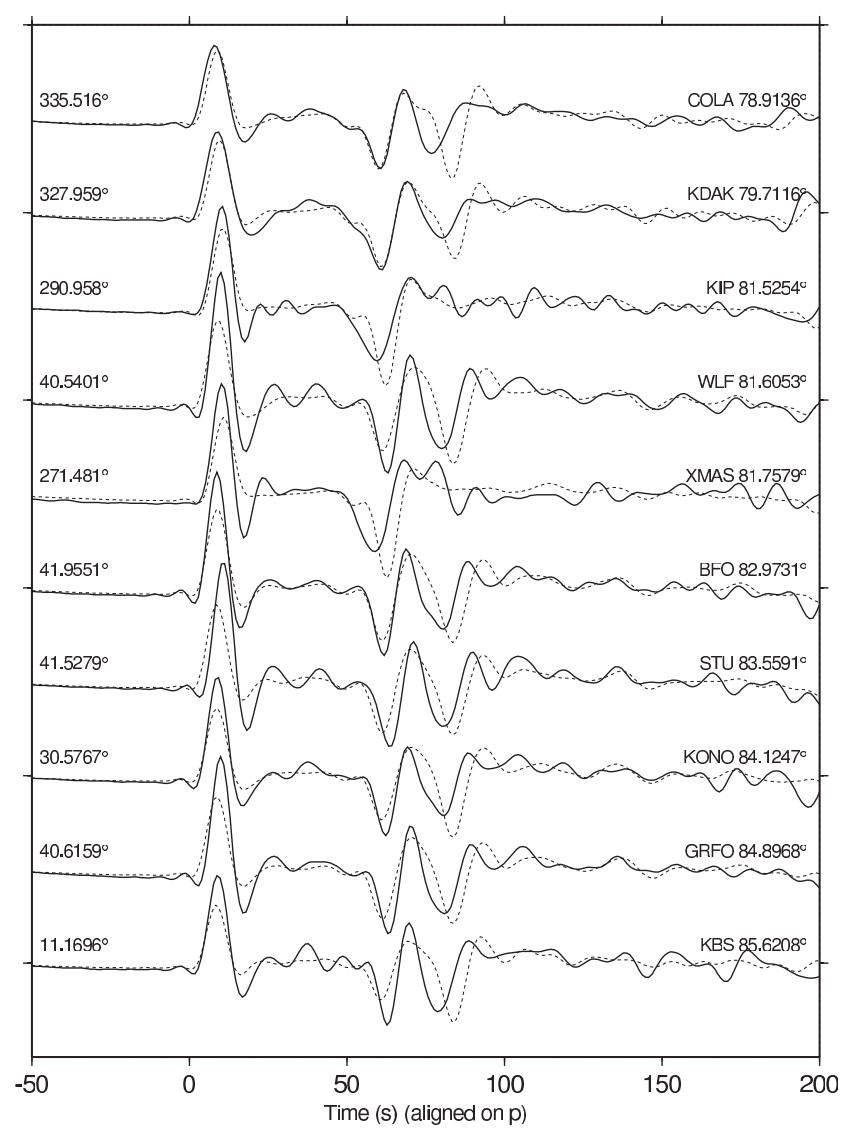

Figure 8. Comparison of vertical component data (solid line) and spectral-element synthetic seismograms (dashed line) for the September 2, 1997, $210 \mathrm{~km}$ deep Colombia earthquake. Both the synthetic seismograms and the data are lowpass-filtered at $5 \mathrm{~s}$. The source azimuth measured clockwise from due North is indicated on the left, and the station name and epicentral distance are on the right. Records are aligned on the $\mathrm{P}$ wave. Note the distinct $\mathrm{pP}$ and $\mathrm{sP}$ arrivals at $60 \mathrm{~s}$ and $80 \mathrm{~s}$ after the $\mathrm{P}$ wave, respectively. The amplitudes in this plot are more than ten times smaller than those in Figure 7.

condition at the CMB or at the ICB, thus making the SEM algorithm much simpler and more efficient numerically. The method has been implemented on a parallel computer with distributed memory based upon a message-passing methodology.

In the current implementation we make the Cowling approximation, make the assumption that the Brunt-Väisälä frequency is zero, use an approximate treatment of the effect of the oceans, and rely on a simple one-way treatment for absorbing boundaries in the case of regional or local simulations. Effects of the Cowling approximation and a nonzero Brunt-Väisälä frequency are only relevant at very long periods (typically $>500 \mathrm{~s}$ ). Improved implementations of 


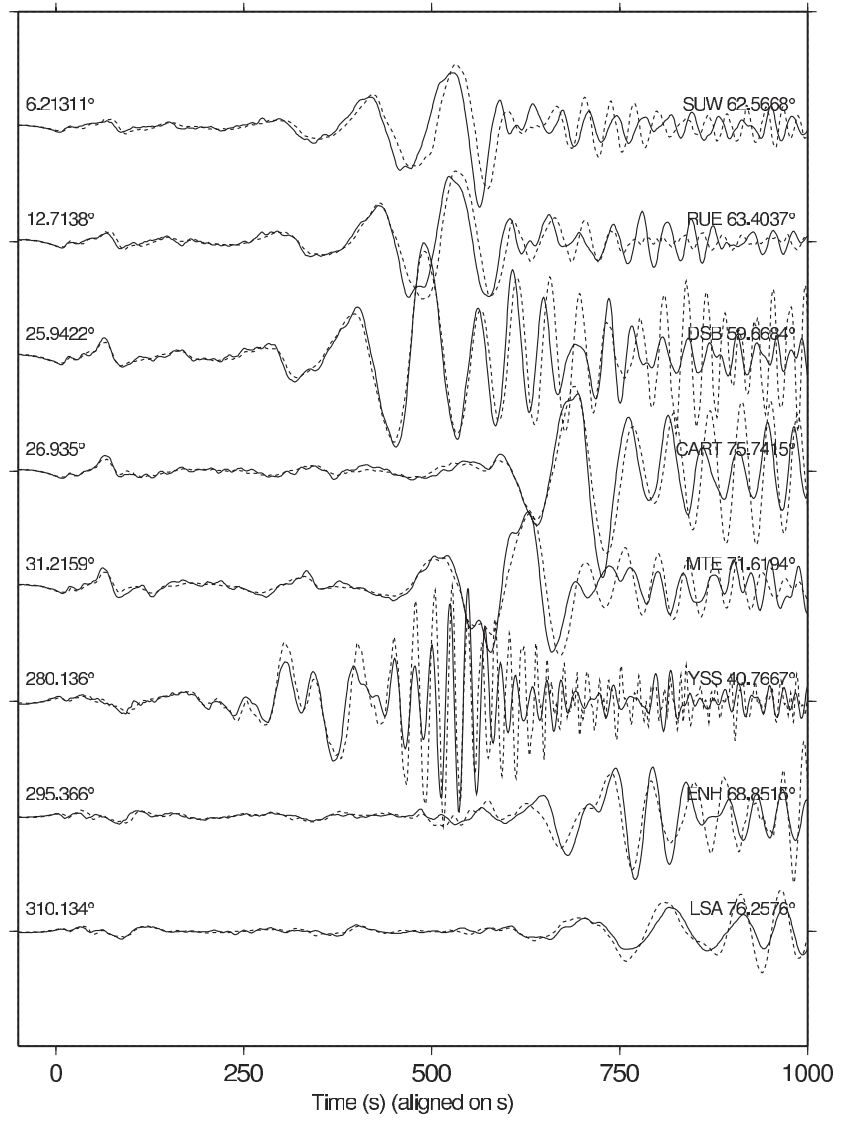

Figure 9. Comparison of vertical component data (solid line) and spectral-element synthetic seismograms (dashed line) for the November 3, 2002, Denali fault, Alaska, earthquake. Both the synthetic seismograms and the data are lowpass-filtered at $5 \mathrm{~s}$. The source azimuth measured clockwise from due North is indicated on the left, and the station name and epicentral distance are on the right. Records are aligned on the $\mathrm{P}$ wave.

the oceans are under investigation and will be the focus of future work.

We have used the Japanese Earth Simulator to perform a direct comparison between synthetic seismograms calculated for a realistic fully 3-D Earth model and observed seismograms for two large earthquakes. This comparison shows that our spectral-element simulations capture general features of the Earth's 3-D structure fairly well. However, it is also apparent that the agreement between synthetic and observed seismograms decreases at high frequency due to the fact that current 3-D Earth models are not well constrained at such high frequencies.

In the near future, we believe that the SEM will become the method of choice for the simulation of seismic wave propagation in fully 3-D Earth models. The main difficulty is the cost of large simulations, as is the case for all methods that are based upon the full wave equation discretized on a grid. However, because of the continuous evolution of computer technology, a small group of researchers can now assemble a PC cluster at a reasonable cost in a reasonable amount of time and perform large-scale 3-D simulations inhouse, and at the same time, very large machines such as the Earth Simulator in Japan or large geographically-distributed networks of clusters of computers (known as GRIDs) start to provide enough memory and processors to run complex models at unprecedented resolution. This has already led to Centroid-Moment Tensor (CMT) source inversion in fully 3-D Earth models [Liu et al., 2004], opening the door to full waveform tomographic inversions.

The full source code of our program SPECFEM3D is freely available for academic, non-commercial research from www.geoframework.org.

Acknowledgments. The authors would like to thank Chen Ji for use of his finite-fault source model for the Denali earthquake. Dimitri Komatitsch also thanks Emmanuel Chaljub, Jean-Pierre Vilotte, Yvon Maday, Anthony T. Patera, Géza Seriani, Enrico Priolo, Fabio Cavallini and Alain Bamberger for enlightening discussions about spectral-element and finite-element methods. The thorough and constructive comments of two anonymous reviewers and editors Alan Levander and Guust Nolet improved the manuscript. Simulations on the Earth Simulator were performed under the collaborative project of the Earth Simulator Center entitled 'Global elastic response simulation'. Broadband data were obtained from the IRIS Data Management Center. This material is based in part upon work supported by the National Science Foundation under grant EAR-0309576. This is contribution No. 9076 of the Division of Geological \& Planetary Sciences, California Institute of Technology, USA.

\section{REFERENCES}

Anderson, D. L., A seismic equation of state, Part II: Shear properties and thermodynamics of the lower mantle, Phys. Earth Planet. Inter., 45, 307-323, 1987.

Bao, H., J. Bielak, O. Ghattas, L. F. Kallivokas, D. R. O'Hallaron, J. R. Shewchuk, and J. Xu, Large-scale simulation of elastic wave propagation in heterogeneous media on parallel computers, Comput. Methods Appl. Mech. Engrg., 152, 85-102, 1998.

Bassin, C., G. Laske, and G. Masters, The current limits of resolution for surface wave tomography in North America, EOS, 81, F897, 2000.

Basu, U., and A. K. Chopra, Perfectly matched layers for transient elastodynamics of unbounded domains, Int. J. Numer. Meth. Engng., 59, 1039-1074, 2004.

Bérenger, J. P., A Perfectly Matched Layer for the absorption of electromagnetic waves, J. Comput. Phys., 114, 185-200, 1994.

Bouchon, M., C. A. Schultz, and M. N. Töksoz, Effect of threedimensional topography on seismic motion, J. Geophys. Res., 101, 5835-5846, 1996. 
Canuto, C., M. Y. Hussaini, A. Quarteroni, and T. A. Zang, Spectral methods in fluid dynamics, Springer-Verlag, New York, 1988.

Capdeville, Y., E. Chaljub, J. P. Vilotte, and J. P. Montagner, Coupling the spectral element method with a modal solution for elastic wave propagation in global Earth models, Geophys. J. Int., 152, 34-67, 2003.

Carcione, J. M., The wave equation in generalized coordinates, Geophysics, 59, 1911-1919, 1994.

Carcione, J. M., D. Kosloff, and R. Kosloff, Wave propagation simulation in a linear viscoelastic medium, Geophys. J. Int., 95, 597-611, 1988.

Chaljub, E., Modélisation numérique de la propagation d'ondes sismiques en géométrie sphérique : application à la sismologie globale (Numerical modeling of the propagation of seismic waves in spherical geometry: application to global seismology), Ph.D. thesis, Université Paris VII Denis Diderot, Paris, France, 2000, in French.

Chaljub, E., and B. Valette, Spectral element modelling of threedimensional wave propagation in a self-gravitating Earth with an arbitrarily stratified outer core, Geophys. J. Int., 158, 131141, 2004.

Chaljub, E., Y. Capdeville, and J. P. Vilotte, Solving elastodynamics in a fluid-solid heterogeneous sphere: a parallel spectral element approximation on non-conforming grids, J. Comput. Phys., 187, 457-491, 2003.

Clayton, R., and B. Engquist, Absorbing boundary conditions for acoustic and elastic wave equations, Bull. Seismol. Soc. Am., 67, 1529-1540, 1977.

Cohen, G., and S. Fauqueux, Mixed finite elements with mass lumping for the transient wave equation, J. Comput. Acoust., 8, 171-188, 2000.

Cohen, G., P. Joly, and N. Tordjman, Construction and analysis of higher-order finite elements with mass lumping for the wave equation, in Proceedings of the second international conference on mathematical and numerical aspects of wave propagation, edited by R. Kleinman, pp. 152-160, SIAM, Philadephia, PA, 1993.

Collino, F., and C. Tsogka, Application of the PML absorbing layer model to the linear elastodynamic problem in anisotropic heterogeneous media, Geophysics, 66, 294-307, 2001.

Courant, R., K. O. Friedrichs, and H. Lewy, Über die partiellen Differenzengleichungen der mathematischen Physik, Mathematische Annalen, 100, 32-74, 1928, in German.

Cowling, T. G., The non-radial oscillations of polytropic stars, Mon. Not. Roy. Astron. Soc., 101, 369-373, 1941.

Dahlen, F. A., and J. Tromp, Theoretical Global Seismology, Princeton University Press, Princeton, 1998.

Day, S. M., Efficient simulation of constant $Q$ using coarse-grained memory variables, Bull. Seismol. Soc. Am., 88, 1051-1062, 1998.

Dziewonski, A. M., and D. L. Anderson, Preliminary reference Earth model, Phys. Earth Planet. Inter., 25, 297-356, 1981.

Eberhart-Phillips, D., et al., The 2002 Denali fault earthquake, Alaska: a large magnitude, slip-partitioned event, Science, 300, 1113-1118, 2003.

Emmerich, H., and M. Korn, Incorporation of attenuation into timedomain computations of seismic wave fields, Geophysics, 52, 1252-1264, 1987.
Faccioli, E., F. Maggio, R. Paolucci, and A. Quarteroni, 2D and 3D elastic wave propagation by a pseudo-spectral domain decomposition method, J. Seismol., 1, 237-251, 1997.

Festa, G., and J. P. Vilotte, The Newmark scheme as velocity-stress time-staggering: an efficient PML implementation for spectral element simulations of elastodynamics, Geophys. J. Int., 161, 789-812, doi:10.1111/j.1365-246X.2005.02601.x, 2005.

Graves, R. W., Simulating seismic wave propagation in 3D elastic media using staggered-grid finite differences, Bull. Seismol. Soc. Am., 86, 1091-1106, 1996.

Gropp, W., E. Lusk, and A. Skjellum, Using MPI, portable parallel programming with the Message-Passing Interface, MIT Press, Cambridge, 1994.

Guo, B., and I. Babuška, The $h-p$ version of the finite element method, Comput. Mech., 1, 21-41, 1986.

Hughes, T. J. R., The finite element method, linear static and dynamic finite element analysis, Prentice-Hall International, Englewood Cliffs, NJ, 1987.

Ji, C., D. V. Helmberger, and D. J. Wald, A teleseismic study of the 2002 Denali fault, Alaska, earthquake and implications for rapid strong-motion estimation, Earthquake Spectra, 20, 617637, 2004.

Karato, S., Importance of anelasticity in the interpretation of seismic tomography, Geophys. Res. Lett., 20, 1623-1626, 1993.

Kawase, H., Time-domain response of a semi-circular canyon for incident $S V, P$ and Rayleigh waves calculated by the discrete wavenumber boundary element method, Bull. Seismol. Soc. Am., 78, 1415-1437, 1988.

Kennett, B. L. N., and E. R. Engdahl, Traveltimes for global earthquake location and phase identification, Geophys. J. Int., 105, 429-465, 1991.

Komatitsch, D., Méthodes spectrales et éléments spectraux pour l'équation de l'élastodynamique $2 \mathrm{D}$ et $3 \mathrm{D}$ en milieu hétérogène (Spectral and spectral-element methods for the 2D and 3D elastodynamics equations in heterogeneous media), Ph.D. thesis, Institut de Physique du Globe, Paris, France, 1997, in French, 187 pages.

Komatitsch, D., and J. Tromp, Introduction to the spectral-element method for 3-D seismic wave propagation, Geophys. J. Int., 139, 806-822, 1999.

Komatitsch, D., and J. Tromp, Spectral-element simulations of global seismic wave propagation-I. Validation, Geophys. J. Int., 149, 390-412, 2002a.

Komatitsch, D., and J. Tromp, Spectral-element simulations of global seismic wave propagation-II. 3-D models, oceans, rotation, and self-gravitation, Geophys. J. Int., 150, 303-318, 2002 b.

Komatitsch, D., and J. Tromp, A Perfectly Matched Layer absorbing boundary condition for the second-order seismic wave equation, Geophys. J. Int., 154, 146-153, 2003.

Komatitsch, D., and J. P. Vilotte, The spectral-element method: an efficient tool to simulate the seismic response of 2D and 3D geological structures, Bull. Seismol. Soc. Am., 88, 368-392, 1998.

Komatitsch, D., C. Barnes, and J. Tromp, Wave propagation near a fluid-solid interface: a spectral element approach, Geophysics, 65, 623-631, 2000a. 
Komatitsch, D., C. Barnes, and J. Tromp, Simulation of anisotropic wave propagation based upon a spectral element method, Geophysics, 65, 1251-1260, 2000b.

Komatitsch, D., R. Martin, J. Tromp, M. A. Taylor, and B. A. Wingate, Wave propagation in 2-D elastic media using a spectral element method with triangles and quadrangles, J. Comput. Acoust., 9, 703-718, 2001.

Komatitsch, D., J. Ritsema, and J. Tromp, The spectral-element method, Beowulf computing, and global seismology, Science, 298, 1737-1742, 2002.

Komatitsch, D., S. Tsuboi, C. Ji, and J. Tromp, A 14.6 billion degrees of freedom, 5 teraflops, 2.5 terabyte earthquake simulation on the Earth Simulator, Proceedings of the ACM/IEEE Supercomputing SC'2003 conference, 2003, published on CDROM and at www.sc-conference.org/sc2003.

Komatitsch, D., Q. Liu, J. Tromp, P. Süss, C. Stidham, and J. H. Shaw, Simulations of ground motion in the Los Angeles basin based upon the spectral-element method, Bull. Seismol. Soc. Am., 94, 187-206, 2004.

Liu, H. P., D. L. Anderson, and H. Kanamori, Velocity dispersion due to anelasticity: implications for seismology and mantle composition, Geophys. J. R. Astron. Soc., 47, 41-58, 1976.

Liu, Q., J. Polet, D. Komatitsch, and J. Tromp, Spectral-element moment-tensor inversions for earthquakes in Southern California, Bull. Seismol. Soc. Am., 94, 1748-1761, 2004.

Madariaga, R., Dynamics of an expanding circular fault, Bull. Seismol. Soc. Am., 65, 163-182, 1976.

Maday, Y., and E. M. Rønquist, Optimal error analysis of spectral methods with emphasis on non-constant coefficients and deformed geometries, Comput. Methods Appl. Mech. Engrg., 80, 91-115, 1990.

Marfurt, K. J., Accuracy of finite-difference and finite-element modeling of the scalar wave equation, Geophysics, 49, 533549, 1984.

Moczo, P., E. Bystrický, J. Kristek, J. M. Carcione, and M. Bouchon, Hybrid modeling of $P-S V$ seismic motion at inhomogeneous viscoelastic topographic structures, Bull. Seismol. Soc. Am., 87, 1305-1323, 1997.

Ohminato, T., and B. A. Chouet, A free-surface boundary condition for including 3D topography in the finite difference method, Bull. Seismol. Soc. Am., 87, 494-515, 1997.

Patera, A. T., A spectral element method for fluid dynamics: laminar flow in a channel expansion, J. Comput. Phys., 54, 468488, 1984.

Priolo, E., J. M. Carcione, and G. Seriani, Numerical simulation of interface waves by high-order spectral modeling techniques, J. Acoust. Soc. Am., 95, 681-693, 1994.

Quarteroni, A., A. Tagliani, and E. Zampieri, Generalized Galerkin approximations of elastic waves with absorbing boundary conditions, Comput. Methods Appl. Mech. Engrg., 163, 323-341, 1998.

Ritsema, J., H. J. Van Heijst, and J. H. Woodhouse, Complex shear velocity structure imaged beneath Africa and Iceland, Science, 286, 1925-1928, 1999.

Robertsson, J. O. A., A numerical free-surface condition for elastic/ viscoelastic finite-difference modeling in the presence of topography, Geophysics, 61, 1921-1934, 1996.
Ronchi, C., R. Ianoco, and P. S. Paolucci, The "Cubed Sphere'" a new method for the solution of partial differential equations in spherical geometry, J. Comput. Phys., 124, 93-114, 1996.

Sadourny, R., Conservative finite-difference approximations of the primitive equations on quasi-uniform spherical grids, Monthly Weather Review, 100, 136-144, 1972.

Sánchez-Sesma, F. J., and M. Campillo, Diffraction of $P, S V$ and Rayleigh waves by topographic features: a boundary integral formulation, Bull. Seismol. Soc. Am., 81, 2234-2253, 1991.

Schubert, B., The spectral-element method for seismic wave propagation: theory, implementation and comparison to finite difference methods, Tech. rep., University of Munich, Germany, 2003, unpublished report, $165 \mathrm{p}$.

Seriani, G., A parallel spectral element method for acoustic wave modeling, J. Comput. Acoust., 5, 53-69, 1997.

Seriani, G., 3-D large-scale wave propagation modeling by a spectral element method on a Cray T3E multiprocessor, Comput. Methods Appl. Mech. Engrg., 164, 235-247, 1998.

Seriani, G., and E. Priolo, A spectral element method for acoustic wave simulation in heterogeneous media, Finite Elements in Analysis and Design, 16, 337-348, 1994.

Sherwin, S. J., and G. E. Karniadakis, A triangular spectral element method: applications to the incompressible Navier-Stokes equations, Comput. Methods Appl. Mech. Engrg., 123, 189-229, 1995.

Taylor, M., J. Tribbia, and M. Iskandarani, The spectral element method for the shallow water equation on the sphere, J. Comput. Phys., 130, 92-108, 1997.

Taylor, M. A., and B. A. Wingate, A generalized diagonal mass matrix spectral element method for non-quadrilateral elements, Appl. Num. Math., 33, 259-265, 2000.

Tessmer, E., D. Kessler, D. Kosloff, and A. Behle, Multi-domain Chebyshev-Fourier method for the solution of the equations of motion of dynamic elasticity, J. Comput. Phys., 100, 355363, 1992.

Tordjman, N., Éléments finis d'ordre élevé avec condensation de masse pour l'équation des ondes (High-order finite elements with mass lumping for the wave equation), Ph.D. thesis, Université Paris IX Dauphine, Paris, France, 1995, in French.

Tsuboi, S., D. Komatitsch, C. Ji, and J. Tromp, Spectral-element simulations of the November 3, 2002, Denali, Alaska earthquake on the Earth Simulator, Phys. Earth Planet. Inter., 139, 305313, 2003.

Valette, B., About the influence of prestress upon the adiabatic perturbations of the Earth, Geophys. J. R. Astron. Soc., 85, 179208, 1986.

Valette, B., Spectre des oscillations libres de la Terre: aspects mathématiques et géophysiques (Spectrum of the free oscillations of the Earth: mathematical and geophysical aspects), Habilitation thesis, Université Paris VI Jussieu, Paris, 1987, thèse d'état, in French.

Virieux, J., $P$-SV wave propagation in heterogeneous media: velocity-stress finite-difference method, Geophysics, 51, 889-901, 1986.

Zeng, X., Finite difference modeling of viscoelastic wave propagation in a generally heterogeneous medium in the time domain, 
and a dissection method in the frequency domain, Ph.D. thesis, University of Toronto, Canada, 1996.

Zienkiewicz, O. C., The finite element method in engineering science, 3rd ed., McGraw-Hill, New York, 1977.

Zingg, D. W., Comparison of high-accuracy finite-difference methods for linear wave propagation, SIAM Journal on Scientific Computing, 22, 476-502, 2000, doi: 10.1137/ S1064827599350320.

Zingg, D. W., H. Lomax, and H. Jurgens, High-accuracy finitedifference schemes for linear wave propagation, SIAM Journal on Scientific Computing, 17, 328-346, 1996, doi: 10.1137/ S1064827594267173.
Dimitri Komatitsch, CNRS FRE 2639, Université de Pau et des Pays de l'Adour, Avenue de l'Université, 64013 Pau Cedex, France. (e-mail: dimitri.komatitsch@univ-pau.fr)

Seiji Tsuboi, Institute for Research on Earth Evolution, Japan Agency for Marine-Earth Science and Technology, 3173-25 Showa-machi, Kanazawa-ku, Yokohama 236-0001, Japan. (e-mail: tsuboi@jamstec.go.jp)

Jeroen Tromp, Seismological Laboratory, 1200 East California Boulevard, California Institute of Technology, Pasadena, California 91125, USA. (e-mail: jtromp@ caltech.edu) 